\title{
A catalogue of dense cores and young stellar objects in the Lupus complex based on Herschel` Gould Belt Survey observations ${ }^{\star \star}$
}

\author{
M. Benedettini ${ }^{1}$, S. Pezzuto ${ }^{1}$, E. Schisano ${ }^{1}$, P. André ${ }^{2}$, V. Könyves ${ }^{3,2}$, A. Men'shchikov ${ }^{2}$, B. Ladjelate $^{4,2}$, \\ J. Di Francesco ${ }^{5}$, D. Elia ${ }^{1}$, D. Arzoumanian ${ }^{6,2}$, F. Louvet ${ }^{2}$, P. Palmeirim ${ }^{7}$, K. L. J. Rygl ${ }^{8}$, N. Schneider ${ }^{9}$, \\ L. Spinoglio ${ }^{1}$, and D. Ward-Thompson ${ }^{3}$
}

${ }^{1}$ INAF - Istituto di Astrofisica e Planetologia Spaziali, via Fosso del Cavaliere 100, 00133 Roma, Italy e-mail: milena.benedettini@inaf.it

${ }^{2}$ Laboratoire AIM, CEA/DSM-CNRS-Université Paris Diderot, IRFU/Service d'Astrophysique, Saclay, 91191 Gif-sur-Yvette, France

3 Jeremiah Horrocks Institute, University of Central Lancashire, Preston PR1 2HE, UK

${ }^{4}$ Instituto Radioastronomía Milimétrica, Av. Divina Pastora 7, Nucleo Central, 18012 Granada, Spain

${ }^{5}$ National Research Council Canada, 5071 West Saanich Road, Victoria, BC V9E 2E7, Canada

${ }^{6}$ Department of Physics, Graduate School of Science, Nagoya University, Furo-cho, Chikusa-ku, Nagoya 464-8602, Japan

${ }^{7}$ Instituto de Astrofísica e Ciências do Espaço, Universidade do Porto, CAUP, Rua das Estrelas, PT4150-762 Porto, Portugal

${ }^{8}$ Italian ALMA Regional Centre, INAF - Istituto di Radioastronomia, via P. Gobetti 101, 40129, Bologna, Italy

${ }^{9}$ I. Physikalisches Institut, Universität zu Köln, Zulpicher Str. 77, 50939 Köln, Germany

Received 4 May 2018 / Accepted 13 August 2018

\begin{abstract}
Context. How the diffuse medium of molecular clouds condenses in dense cores and how many of these cores will evolve in protostars is still a poorly understood step of the star formation process. Much progress is being made in this field, thanks to the extensive imaging of star-forming regions carried out with the Herschel Space Observatory.

Aims. The Herschel Gould Belt Survey key project mapped the bulk of nearby star-forming molecular clouds in five far-infrared bands with the aim of compiling complete census of prestellar cores and young, embedded protostars. From the complete sample of prestellar cores, we aim at defining the core mass function and studying its relationship with the stellar initial mass function. Young stellar objects (YSOs) with a residual circumstellar envelope are also detected.

Methods. In this paper, we present the catalogue of the dense cores and YSOs/protostars extracted from the Herschel maps of the Lupus I, III, and IV molecular clouds. The physical properties of the detected objects were derived by fitting their spectral energy distributions.

Results. A total of 532 dense cores, out of which 103 are presumably prestellar in nature, and 38 YSOs/protostars have been detected in the three clouds. Almost all the prestellar cores are associated with filaments against only about one third of the unbound cores and YSOs/protostars. Prestellar core candidates are found even in filaments that are on average thermally subcritical and over a background column density lower than that measured in other star-forming regions so far. The core mass function of the prestellar cores peaks between 0.2 and $0.3 M_{\odot}$, and it is compatible with the log-normal shape found in other regions. Herschel data reveal several, previously undetected, protostars and new candidates of Class 0 and Class II with transitional disks. We estimate the evolutionary status of the YSOs/protostars using two independent indicators: the $\alpha$ index and the fitting of the spectral energy distribution from near- to farinfrared wavelengths. For $70 \%$ of the objects, the evolutionary stages derived with the two methods are in agreement.

Conclusions. Lupus is confirmed to be a very low-mass star-forming region, in terms of both the prestellar condensations and the diffuse medium. Noticeably, in the Lupus clouds we have found star formation activity associated with interstellar medium at low column density, usually quiescent in other (more massive) star-forming regions.
\end{abstract}

Key words. ISM: clouds - ISM: individual objects: Lupus complex (except planetary nebulae) - stars: formation submillimeter: ISM

\section{Introduction}

Stars form in the denser filamentary regions of Giant Molecular Clouds (GMCs) but how diffuse matter gathers in cores

\footnotetext{
${ }^{\star}$ Herschel is an ESA space observatory with science instruments provided by European-led Principal Investigator consortia and with important participation from NASA.

${ }^{\star \star}$ Full Tables A.1 and A.2 are only available at the CDS via anonymous ftp to cdsarc.u-strasbg.fr (130.79.128.5) or via http://cdsarc.u-strasbg.fr/viz-bin/qcat?J/A+A/619/A52
}

and how many of these dense cores will form stars is still a poorly understood process. To provide insight into the physical mechanisms responsible for the growth of structure in the cold interstellar medium (ISM), leading to the formation of prestellar cores and protostars is one of the main scientific goals of the Herschel Gould Belt Survey (HGBS) key project that mapped the main nearby $(d \lesssim 500 \mathrm{pc})$ star-forming regions in five farinfrared (FIR) bands with Herschel's photometric instruments (André et al. 2010). The main products of the HGBS project are comprehensive and homogeneous catalogues of compact sources 
for all the observed regions. In this paper, we present catalogues of dense cores and young stellar objects (YSOs) extracted from the Herschel observations of the star-forming regions in the Lupus complex and we derive their physical properties.

The Lupus dark cloud complex is located in the ScorpiusCentaurus OB association and consists of several loosely connected dark clouds showing different levels of star formation activity. With Herschel, we mapped the three main sites of star formation within the complex, namely the Lupus I, Lupus III, and Lupus IV clouds. These three clouds have been intensively observed with other instruments at several wavelengths to study the star formation process in the low-mass regime. The YSOs population in these clouds was initially investigated at nearinfrared (NIR) and mid-infrared (MIR) wavelengths as part of the Spitzer-c2d survey (Merin et al. 2008). The Lupus I cloud was observed also at 450 and $850 \mu \mathrm{m}$ as part of the James Clerk Maxwell Telescope Gould Belt Survey (Mowat et al. 2017), revealing a number of cold and dense condensations. Fifteen of these were identified as disks of YSOs and 12 as prestellar or protostellar cores. Emission line maps from high-density molecular tracers at 3 and $12 \mathrm{~mm}$ with the Mopra telescope have allowed to be made a chemical classification of the brighter and denser cores from which indications of their evolutionary status were inferred (Benedettini et al. 2012).

In Benedettini et al. (2015; hereafter Paper I), we presented the analysis of the filamentary structure of the clouds extracted from the Herschel-based column density maps. This study has revealed that Lupus I, III, and IV clouds have an ISM characterised by a very low column density in both the diffuse regions and the dense material arranged in filaments. Indeed, the probability distribution function of column density $(\mathrm{PDF})$ in the three regions peaks between $5 \times 10^{20}$ and $5 \times 10^{21} \mathrm{~cm}^{-2}$, and the average column density of filaments is only $\sim 1.5 \times 10^{21} \mathrm{~cm}^{-2}$ (Paper I). The absence of very high column density gas $\left(\geq 5 \times 10^{22} \mathrm{~cm}^{-2}\right)$ and the predominance of low column density gas likely has an impact on the typical mass of stars formed in these regions. Lupus is indeed a low-mass starforming complex with a stellar population dominated by mid M-type stars (Hughes et al. 1994; Mortier et al. 2011; Alcalá et al. 2017). A preliminary visual version of a catalogue of dense cores based on Herschel data was presented in Rygl et al. (2013).

The Lupus complex is one of the closer sites of low-mass star formation, but the precise distances of its subregions is still a matter of debate. Recently, Galli et al. (2013) investigated the kinematic properties of the Lupus moving group of young stars and derived the following distances: $d=182_{-6}^{+7}$ pc for Lupus I, $d=185_{-10}^{+11}$ pc for Lupus III, and $d=204_{-15}^{+18}$ pc for Lupus IV. We derived distances from the Gaia DR 2 paralaxes (Luri et al. 2018) for a sample of know YSOs members of the Lupus complex and we found the following modal values: $d=155_{-14}^{+7} \mathrm{pc}$ for Lupus I, $d=160_{-8}^{+45} \mathrm{pc}$ for Lupus III, and $d=155_{-18}^{+30} \mathrm{pc}$ for Lupus IV, where the negative and positive errors correspond to the minimum $5 \%$ and maximum $95 \%$ quantile of all the quantiles of the objects in each of the three clouds. Previous distance estimates, based also on different methods, give values between 140 and $200 \mathrm{pc}$ for the different members of the complex. In this work, we assume the values indicated by Comerón (2008) who reviewed all the distance estimates in the previous literature, concluding that a distance of $150 \mathrm{pc}$ seems adequate for Lupus I and IV, while a value of $200 \mathrm{pc}$ is more appropriate for Lupus III. The same distances were used also in the Spitzer-c2d catalogue of YSOs (Merin et al. 2008) and in Paper I and they are within the errors associated with the values derived by Gaia DR 2 data.
In this paper we present the catalogue of the dense cores and YSOs/protostars extracted from the Herschel maps of Lupus I, III, and IV. In Sect. 2 we describe the observations and data products. The procedures for compact source and protostar extraction and selection are presented in Sect. 3. In Sects. 4 and 5, we present the catalogues of dense cores and YSOs/protostars, respectively, and how the physical properties of the objects in the catalogues are derived. The analysis of the main properties of the objects of the two catalogues is discussed in Sect. 6. The main conclusions of the paper are summarised in Sect. 7.

\section{Observations and data reduction}

As part of the $\mathrm{HGBS}^{1}$ (André et al. 2010), the three subregions of the Lupus complex, Lupus I, III, and IV, were observed in five photometric bands between 70 and $500 \mu \mathrm{m}$ with the Photodetector Array Camera and Spectrometer (PACS; Poglitsch et al. 2010) and the Spectral and Photometric Imaging Receiver (SPIRE; Griffin et al. 2010) onboard the Herschel Space Observatory (Pilbratt et al. 2010). The observations were carried out in the parallel observing mode with a scanning velocity of $60^{\prime \prime} \mathrm{s}^{-1}$ and each cloud was observed twice along orthogonal scanning directions.

The data reduction pipeline and the final maps were presented in Paper I to which we refer for details. Here we recall that the final maps, in unit of $\mathrm{MJy} \mathrm{sr}^{-1}$, have a pixel size $3^{\prime \prime}$ at 70 and $160 \mu \mathrm{m}, 6^{\prime \prime}$ at $250 \mu \mathrm{m}, 10^{\prime \prime}$ at $350 \mu \mathrm{m}$, and $14^{\prime \prime}$ at $500 \mu \mathrm{m}$. The absolute flux calibration for point sources is $\leq 6 \%$ for PACS (Nielbock et al. 2013) and $\leq 5 \%$ for SPIRE (Bendo et al. 2013). The extended sources calibration is more uncertain and we assume a conservative error of $20 \%$. For both PACS and SPIRE maps, we added a zero-level offset derived by comparing the Herschel data with the Planck and IRAS data of the same area of the sky and adopting a dust model for extrapolating the flux at the Herschel wavelengths (Bernard et al. 2010). The calibrated maps were used to produce an $\mathrm{H}_{2}$ column density map at the higher resolution of the SPIRE $250 \mu \mathrm{m}$ data of $188^{\prime \prime} 2$, by applying a method based on a multi-scale decomposition of the data (Palmeirim et al. 2013) and a dust opacity law of $\kappa_{\lambda}=$ $\kappa_{300}(\lambda / 300 \mu \mathrm{m})^{-\beta}$ with $\kappa_{300}=0.1 \mathrm{~cm}^{2} \mathrm{~g}^{-1}$ (already accounting for a gas-to-dust ratio of 100), a grain emissivity parameter $\beta=2$ (Hildebrand 1983), and a mean molecular weight $\mu=2.8$, values adopted as the standard by the HGBS consortium. The five Herschel maps, as well as the high-resolution $\mathrm{H}_{2}$ column density map, for the three clouds are available in the HGBS archive ${ }^{2}$.

Notably, the Lupus I maps were affected by stray moonlight, visible as a bright vertical band in each image. This problem does not affect the estimates of the point sources fluxes since those are measured by applying a background subtraction, but it required correction before the derivation of the column density map. We removed the stray-light contamination in the four images at $160,250,350$, and $500 \mu \mathrm{m}$, by evaluating its contribution from the difference between the observed map (affected by stray-light) and the model used for the flux calibration, producing a column density map corrected from the stray-light effect.

\section{Compact sources identification}

\subsection{Source classification}

The wavelength range covered by the Herschel photometric instruments is where the thermal emission of cold dust

\footnotetext{
1 http://gouldbelt-herschel.cea.fr

2 http://gouldbelt-herschel.cea.fr/archives
} 
( $T \approx 10-30 \mathrm{~K})$ peaks. Therefore, the cold dust condensations which may potentially form stars are clearly visible as compact, roundish structures that emerge from the background in the Herschel maps and in the $\mathrm{H}_{2}$ column density maps. Moreover, in the nearest molecular clouds as those of the Lupus complex, the extremely sensitive Herschel instruments were able to detect even the fainter cores. Most of them are not dense enough to undergo gravitational collapse and will dissipate. Finally, circumstellar envelope of YSOs are detectable in Herschel maps.

To classify the compact sources extracted from the Herschel maps we used the following scheme (see also André et al. 2014). The detection in the PACS $70 \mu \mathrm{m}$ map was used to identify the YSOs/protostars since the presence inside the cores of an accreting object produces an internal heating that increments the emission at wavelengths $\lesssim 100 \mu \mathrm{m}$ with respect to grey-body SED shape, typical of prestallar cores. In fact, the PACS $70 \mu \mathrm{m}$ sensitivity is adequate to detect YSOs emission even in low-luminosity sources but not that of grey-body emitters with temperature around $10 \mathrm{~K}$. Sources without the $70 \mu \mathrm{m}$ detection are considered starless dense cores and are divided into two groups: the gravitationally unbound cores, which are expected to dissipate in the future, and the gravitationally bound cores, which are considered good candidates prestellar cores. To establish if a starless core is gravitationally bound or unbound, we used the ratio between its observed mass and the BonnorEbert mass calculated by using the core measured radius and temperature. More details are given in Sect. 4.2.

\subsection{Sources extraction from Herschel maps}

To generate an extensive catalogue of dense condensations in the Lupus regions, we used the strategy developed inside the HGBS consortium to ensure homogeneous data analysis among all the star-forming regions observed in the project (Könyves et al. 2015). SPIRE and PACS images were processed with getsources $\mathrm{v} 1.140127$, an algorithm that performs the extraction of compact sources and filamentary structures at a multi-scale and multi-wavelength level (Men'shchikov et al. 2012; Men'shchikov 2013). The source extraction method is divided into two stages: a first "detection" stage and a second "measurement" stage. At the detection stage, getsources analyses "single-scale" images across a wide range of scales and across all observed wavebands, identifying the sources and their footprints. At the measurement stage, fluxes and sizes of detected sources are measured in the observed images at each wavelength, considering deblending of overlapping sources. Background is subtracted by linear interpolation under the source footprints found at the detection stage, constrained by different angular resolutions in each waveband. Aperture corrections are applied by getsources using tables of the encircled energy fraction values for the actual point spread functions provided by the PACS and SPIRE ICCs (Bendo et al. 2013; Balog et al. 2014).

Two different getsources extractions are performed, optimised for the detection of dense cores and protostars, respectively. In the first set, we used the Herschel maps at 160, 250, 350, and $500 \mu \mathrm{m}$. In addition, we included the high-resolution column density image - as an additional "wavelength" - to ensure that detected sources correspond to genuine column density peaks. Furthermore, the $160 \mu \mathrm{m}$ component at the detection stage is "temperature-corrected" to reduce the effects of anisotropic temperature gradients. The temperature-corrected $160 \mu \mathrm{m}$ map is obtained by converting the original observed $160 \mu \mathrm{m}$ map (13". 5 resolution) to an approximate column density image, using the colour-temperature map derived from the intensity ratio between 160 and $250 \mu \mathrm{m}$ (at the 18 ". 2 resolution of the $250 \mu \mathrm{m}$ map). The second set of getsources extractions is optimised to detect YSOs/protostars. In this case, at the detection stage we used only the $70 \mu \mathrm{m}$ Herschel image. Indeed, the presence of point-like $70 \mu \mathrm{m}$ emission is a strong indication of the presence of a protostar that is warming up its circumstellar envelope.

At the measurement stage of both sets of extractions, source properties are measured at the detected positions of either cores or YSOs/protostars, using the observed, background-subtracted, and deblended images at all five Herschel wavelengths, plus the high-resolution column density map. The advantage of this two-pronged extraction strategy is that it provides more reliable detections and measurements of column-density cores and $70 \mu \mathrm{m}$ luminous YSOs/protostars, respectively.

\subsection{Selection criteria}

We filtered the raw source lists of the getsources extractions to select only reliable sources by applying the following criteria. These selection criteria are uniformly applied to all the catalogues produced in the HGBS consortium and are described in detail in Könyves et al. (2015).

For candidate dense cores identified from the first set of extractions selection criteria are as follows:

- column density detection significance greater than 5 in the high-resolution column density map;

- global detection significance over all wavelengths greater than 10 ;

- global goodness $\geqslant 1$, where goodness is an output quality parameter of getsources, combining global signal-to-noise ratio $(\mathrm{S} / \mathrm{N})$ and source reliability;

- column density measurement $S / N>1$ in the high-resolution column density map;

- monochromatic detection significance greater than 5 in at least two bands between 160 and $500 \mu \mathrm{m}$;

- flux measurement with $S / N>1$ in at least one band between 160 and $500 \mu \mathrm{m}$ for which the monochromatic detection significance is simultaneously greater than 5 .

For candidate YSOs and protostars identified from the second set of extractions selection criteria are as follows:

- monochromatic detection significance greater than 5 in the $70 \mu \mathrm{m}$ band;

- positive peak and integrated flux densities at $70 \mu \mathrm{m}$;

- global goodness greater than or equal to 1 ;

- flux measurement with $S / N>1.5$ in the $70 \mu \mathrm{m}$ band;

- full width half maximum (FWHM) source size at $70 \mu \mathrm{m}$ smaller than 1.6 times the $70 \mu \mathrm{m}$ beam size (i.e. $<1.6 \times 8$ ". $4=13$ ". 44 );

- estimated source elongation $<1.30$ at $70 \mu \mathrm{m}$, where source elongation is defined as the ratio of the major and minor FWHM sizes.

We also include in our final catalogues three well-known YSOs that were initially present in the raw list, but were successively excluded by the automatic selection based on the above criteria. In principle, some of the sources of our catalogue could be galaxies. To reduce extragalatic contamination as much as possible, we removed any source located within $6^{\prime \prime}$ of known galaxies found by the NASA Extragalactic Database ${ }^{3}$ (NED) and the SIMBAD $^{4}$ database.

We performed a final visual inspection of the selected sample to remove any dubious or not clearly visible source to produce

\footnotetext{
https://ned.ipac.caltech.edu/forms/nearposn.html

4 http://simbad.u-strasbg.fr/simbad/
} 
A\&A 619, A52 (2018)

Table 1. Summary of the number of entries in the catalogues divided for classification and regions.

\begin{tabular}{lcccc}
\hline \hline Region & Total number of objects & Unbound cores & Prestellar cores (robust) & YSOs/protostars \\
\hline Lupus I & 328 & 265 & $54(24)$ & 9 \\
Lupus III & 100 & 59 & $25(19)$ & 16 \\
Lupus IV & 142 & 105 & $24(16)$ & 13 \\
Total & 570 & 429 & $103(59)$ & 38 \\
\hline
\end{tabular}

the final catalogues of cores and YSOs/protostars. These catalogues are available at the CDS with few example lines listed in Table A.1. In these tables, we report all photometric and geometrical quantities measured by getsources in the Herschel maps: position, flux densities, and sizes with their respective errors and also associations with known sources. In Table 1, we summarised the numbers of objects present in the catalogues for the three Lupus clouds, and in Figs. 1-3 we show the sources position on the $250 \mu \mathrm{m}$ maps.

Disentangling the emission of a compact source from the emission of the underlying background in the Herschel maps is not a trivial task because of the usually bright and nonuniform background. Indeed, several algorithms, based on different approaches, have been developed to this purpose. Hence, to provide additional confidence of the reliability of our catalogues, we used a second method to identify compact sources. Namely, we processed the Herschel maps with the Curvature Thresholding EXtractor (CuTEx) algorithm (Molinari et al. 2011). CuTEx extracts compact sources independently on images at each wavelength band. Therefore, we searched for positional matches between our getsources sources and each single-band CuTEx catalogue within the ellipse that defines the size of the source at that band. For the cores catalogue, we consider confirmed matches those that coincide with a CuTEx detection in at least three distinct bands. With this approach, $43 \%$ of the getsources cores were also found by CuTEx. Very similar percentages of cross matches were found in Aquila and Taurus (Könyves et al. 2015; Marsh et al. 2016) when comparing their getsources catalogues with the results from an alternative core extractor such as the Cardiff Sourcefinding AlgoRithm (CSAR; Kirk et al. 2013). For the YSOs/protostars catalogue, we consider a matched source, one that has been detected with CuTEx in the $70 \mu \mathrm{m}$ band. For this class of objects, the percentage of correspondence is much higher, between $85 \%$ and $94 \%$ for the three Lupus regions. This improvement occurs at $70 \mu \mathrm{m}$ because at this wavelength in Lupus the background sky is almost dark while protostars are quite bright and well defined, making the detection of such sources easier. In Table A.1, we supply a robustness flag indicating if the getsources source was also found with the CuTEx algorithm.

\section{Dense cores catalogue}

\subsection{Physical properties}

For the starless dense cores, we estimate the mass and line-ofsight-averaged dust temperature by fitting a modified black-body function to the observed spectral energy distribution (SED) constructed from the set of measured flux densities in the wavelength range $160-500 \mu \mathrm{m}$, weighted by $1 / \sigma^{2} \times$ SIG_MON, where $\sigma$ corresponds to the flux error and SIG_MON is the monochromatic detection significance parameter as estimated by getsources. The dust opacity law is the same as that used for the column density calculation (see Sect. 2). We carried out SED fitting for those dense cores where (i) the monochromatic detection significance is greater than five and the $\mathrm{S} / \mathrm{N}$ of the flux is greater than 0.1 in at least three wavelengths between 160 and $500 \mu \mathrm{m}$; and (ii) the source has a larger integrated flux density at $350 \mu \mathrm{m}$ than at $500 \mu \mathrm{m}$.

In total $86 \%$ of starless cores fulfil the above criteria and we consider their SEDs fitting to be reliable. For the rest of the cores, the masses were directly estimated from the measured integrated flux density at the longest wavelength with a significant detection in each case, assuming optically thin dust emission and the median dust temperature found for starless cores with reliable SED fits (i.e. 11.5 K for Lupus I, 9.6 K for Lupus III, and $11.6 \mathrm{~K}$ for Lupus IV). The corresponding cores are marked as having "no SED fits" in the last column of Table A.2 and they have more uncertain properties with respect to sources for which the fit was performed. The derived physical parameters for each starless dense core are reported in tables available at the CDS, with few example lines listed in Table A.2. In our catalogue, we provide the observed size of the core as the geometrical average between the major and minor FWHM sizes measured in the highresolution column density map and we estimate the core outer radius as the observed size deconvolved with the HPBW of the map (18'.2). The peak (or central beam) column density, the average column density, the central-beam volume density, and the average volume density were also derived for each core based on its estimated mass and radius.

The physical parameters derived from the SED fitting, namely the line-of-sight-averaged dust temperature and mass, are affected by some sources of uncertainty: (i) the error of the flux points due to the absolute flux calibration $(\leq 20 \%)$, the irregular background that could not be well subtracted, and the sources deblending that could be incorrect especially in crowed regions; (ii) the uncertainty of the distances that could be up to $30 \%$ (see Sect. 1); (iii) the uncertainty related to the assumption in the opacity law that is $<50 \%$ for cores in the $\mathrm{H}_{2}$ column density range between $\sim 3 \times 10^{21}$ and $\sim 10^{23} \mathrm{~cm}^{-2}$ (Roy et al. 2014) but could be as high as a factor of 2 for lower $\mathrm{H}_{2}$ column density (Paper I) as those we find in many of the Lupus cores; and (iv) the assumption of single temperatures while prestellar cores have temperature profiles that drop towards their centres. In particular, the temperature profile of the starless dense cores is expected to have a minimum around $9-10 \mathrm{~K}$ at the core centre, where most of the core mass resides, and to increase up to 12-13 K at the core outer radius (e.g. Tafalla et al. 2004; Roy et al. 2014). The mean value of temperature derived from the SED fitting in our catalogue is $\sim 11.5 \mathrm{~K}$. Therefore, the average SED dust temperature may overestimate the mass-averaged dust temperature within a dense core, leading to an underestimate of the core mass. This effect, however, decreases with the decreasing of the core mass, becoming less than $30 \%$ for core masses less than $1 M_{\odot}$ (Men'shchikov 2016). To quantify the cumulative effect of all these factors on the final mass estimate is really complex and it is beyond the scope of the present paper. The simulation that we used for estimating the completeness of the 
M. Benedettini et al.: A catalogue of dense cores and young stellar objects in the Lupus complex

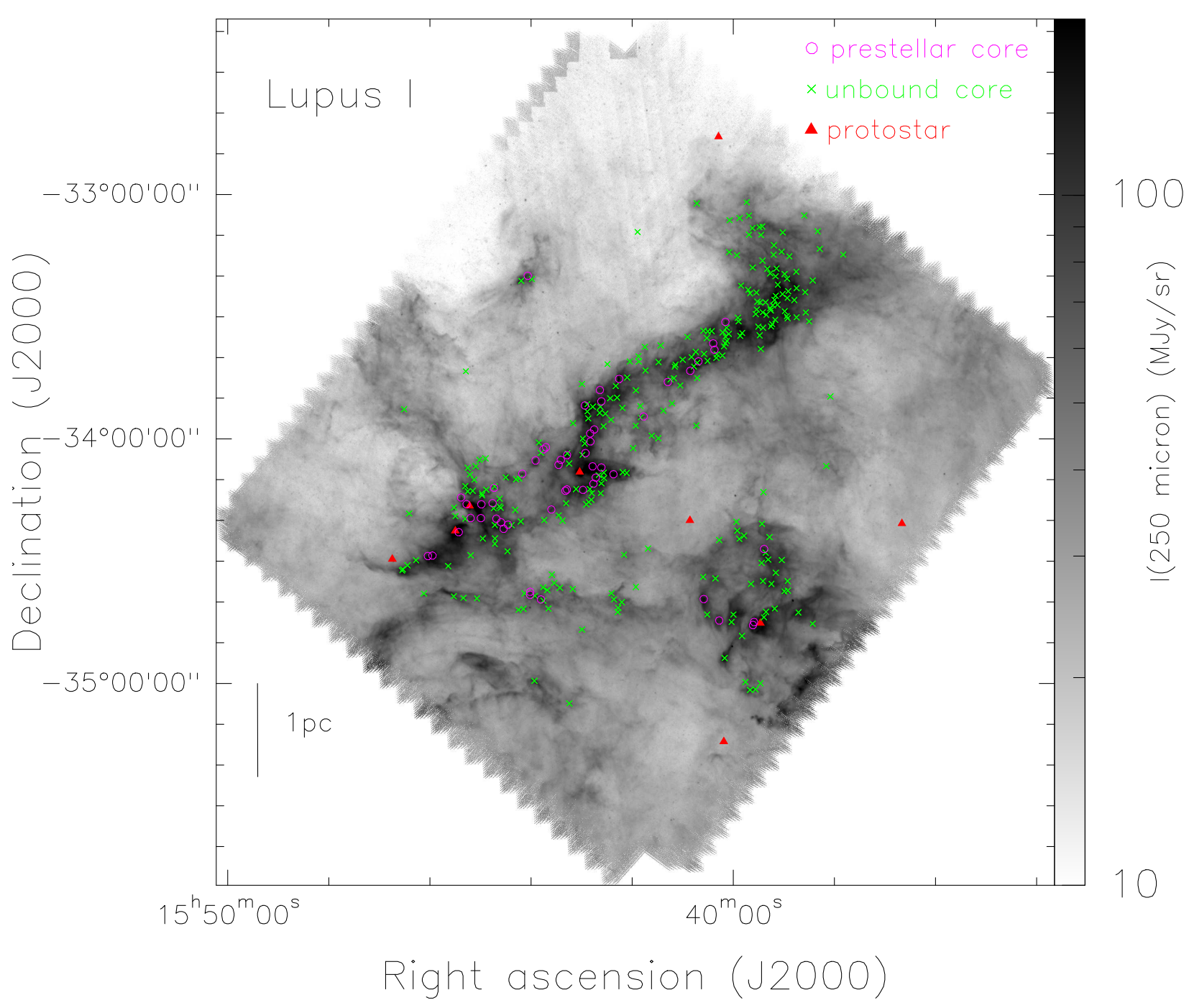

Fig. 1. Herschel $250 \mu \mathrm{m}$ surface brightness image of the Lupus I region. The selected sample of starless unbound cores (crosses), prestellar cores (circles), and protostars (triangles) are indicated.

prestellar cores sample (see Sect. 4.3), however, can help give an idea of the typical error on the mass derived by our sources extraction and SED fitting procedures. We found that the distribution of ratios between the mass derived by the SED fitting and the true core mass can be approximated by a Gaussian function centred around one and with $\sigma \sim 0.4$. This implies that our strongest approximation of single temperature within the cores does not produce systematic underestimates of the masses, in the low mass regime of the Lupus prestellar cores.

\subsection{Selecting self-gravitating prestellar cores}

In the sample of starless cores, we are interested in identifying those cores that are dense enough to eventually form a star or a multiple system, namely the prestellar cores. These objects must be starless and gravitationally bound. For a self-gravitating core, the ratio between its virial mass and its measured mass is $M_{\text {vir }} / M_{\text {obs }} \leq 2$. The virial mass can be derived by measuring the mean velocity dispersion of the gas from spectroscopic observations. Spectroscopic observations, at a spatial resolution similar to that of the Herschel maps, however, are usually unavailable for all the dense cores detected in the Herschel maps. Therefore, a simplified approach is adopted. We look at the ratio between the core mass and the thermal value of the critical Bonnor-Ebert (BE) mass, $\alpha_{\mathrm{BE}}=M_{\mathrm{BE} \text {,crit }} / M_{\mathrm{obs}}$, where the critical Bonnor-Ebert mass is given by $M_{\mathrm{BE}, \mathrm{crit}}=2.4 R_{\mathrm{BE}} c_{\mathrm{s}}^{2} / G$ with $R_{\mathrm{BE}}$ being the BE radius, $G$ being the gravitational constant, and $c_{\mathrm{s}}=\sqrt{k T / \mu m_{\mathrm{H}}}$ being the isothermal sound speed where the non-thermal component of the velocity dispersion is neglected, with $k$ being the Boltzmann's constant, $T$ being the temperature, $\mu=2.33$ being the molecular weigh per particle, and $m_{\mathrm{H}}$ being the hydrogen mass. To get a rough estimate of the contribution of the non-thermal velocity dispersion to the total velocity dispersion that we are neglecting, we used the FWHM of the CS (1-0) line observed towards several dense cores in the Lupus clouds (Benedettini et al. 2012). These cores are larger than those in our catalogue and have a total velocity dispersion ranging from 0.25 to $0.45 \mathrm{~km} \mathrm{~s}^{-1}$, assuming a temperature of $10 \mathrm{~K}$. Since the thermal component of the velocity dispersion at $10 \mathrm{~K}$ is $0.19 \mathrm{~km} \mathrm{~s}^{-1}$, we expect that $M_{\mathrm{BE}, \text { crit }}$ underestimates the $M_{\mathrm{vir}}$ by at most a factor of about 2. For each object, we estimated the thermal BE mass by using as gas temperature the dust temperature estimated by the SED fitting and $R_{\mathrm{BE}}$ as the deconvolved core radius given in Table A.2.

In line with the virial criterion, we classified a prestellar core as "robust" if $\alpha_{\mathrm{BE}}=M_{\mathrm{BE} \text {,crit }} / M_{\mathrm{obs}} \leq 2$. However, previous 


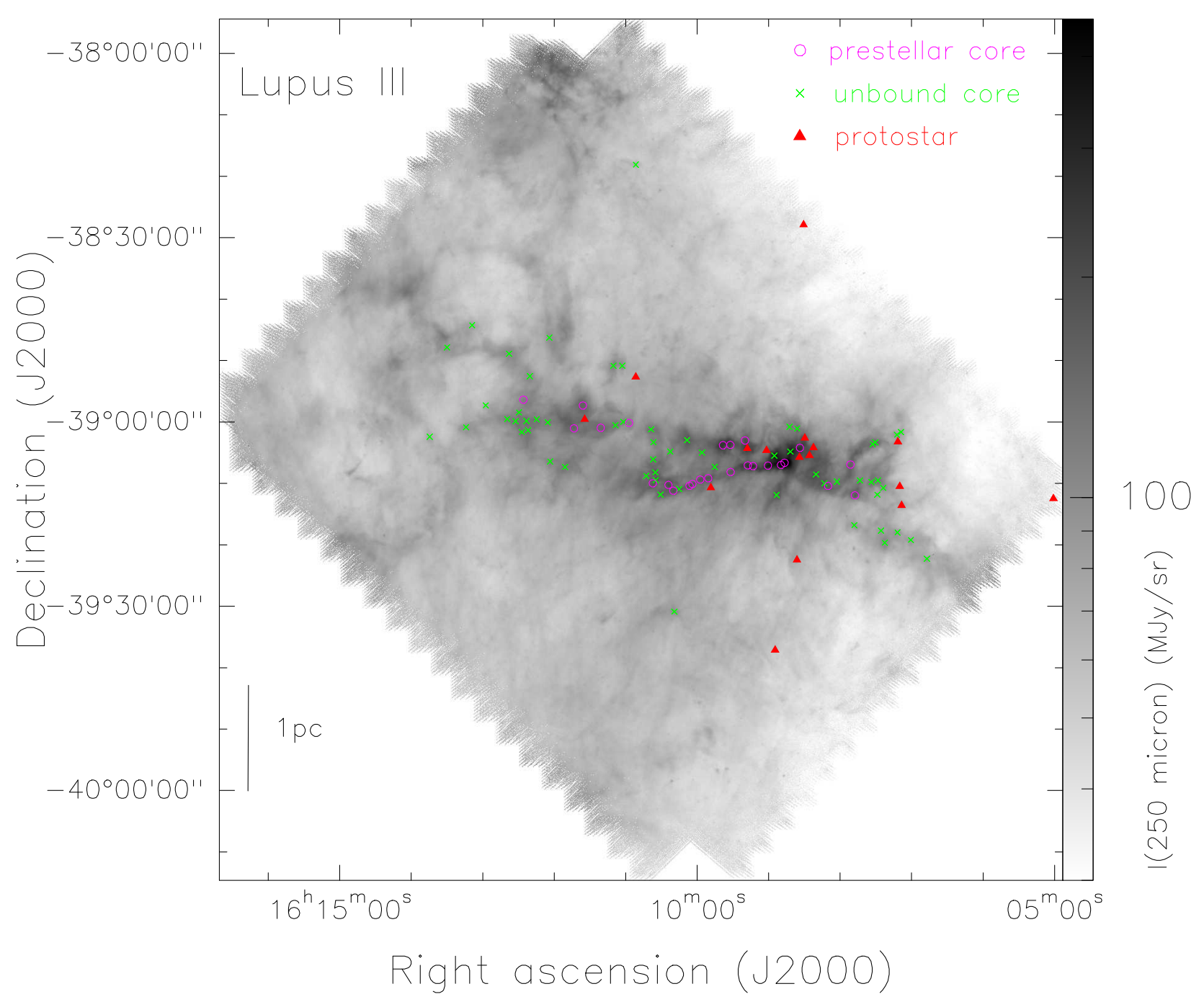

Fig. 2. Herschel $250 \mu \mathrm{m}$ surface brightness image of the Lupus III region. The selected sample of starless unbound cores (crosses), prestellar cores (circles), and protostars (triangles) are indicated.

papers of the HGBS consortium (Könyves et al. 2015; Marsh et al. 2016) have shown that a $\alpha_{\mathrm{BE}}=2$ criterion is too restrictive for selecting gravitationally bound cores. It causes many marginally resolved sources to be misclassified since the critical BE mass depends linearly on radius. To take this effect into consideration, we assume a size-dependent limit $\alpha_{\mathrm{BE}} \leq 5 \times$ $\left(\mathrm{HPBW}_{\mathrm{NH}_{2}} / F W H M_{\mathrm{NH}_{2}}\right)^{0.4}$, where $F W H M_{\mathrm{NH}_{2}}$ is the measured FWHM source diameter in the high-resolution column density map and HPBW $\mathrm{NH}_{2}=18^{\prime \prime} .2$ is the HPBW resolution of the map. In this case, the limiting $\alpha_{\mathrm{BE}}$ ranges from $\sim 2$ for well-resolved cores to $\sim 5$ for unresolved cores. Monte Carlo simulations performed on the Herschel data of the Aquila region have shown that the size-dependent limit allows about $95 \%$ of the prestellar cores to be identified (Könyves et al. 2015).

Following the above criteria, we identify a total of 103 candidate prestellar cores in the three Lupus regions out of which 59 cores can be considered robust candidates. All the rest of the starless cores are classified as unbound cores. The goodness of our classification can be easily assessed by looking at the mass versus radius plot, shown in Fig. 4. As expected, the unbound cores populate the lower right part of the plot, while the candidate prestellar cores occupy the upper left part. In particular, the robust candidate prestellar cores are those above the solid line. We note that the cores of the three Lupus clouds share a very similar range of physical properties, indicating that they form a sample of similar objects. Therefore, we merged the cores catalogues of the three Lupus clouds and applied the following analysis to the merged catalogue.

The percentage of prestellar cores with respect to the total number of starless cores is $19 \%$, in line with that found in Taurus (Marsh et al. 2016), another nearby low-mass starforming region, but lower than that found in more distant and massive star-forming regions. Looking at some of the starforming regions observed by Herschel in the HGBS and HOBYS (Herschel imaging survey of OB Young Stellar objects) key projects, we note that the percentage of prestellar cores with respect to the total number of detected starless cores increases with the distance of the region. For example, it is $\sim 60 \%$ in Aquila (Könyves et al. 2015) at a distance of $d=260$ pc (Cambrésy 1999), $\sim 84 \%$ in Orion (Polychroni et al. 2013) at $d=414 \mathrm{pc}$ (Menten et al. 2016), 94\% in Vela C (Giannini et al. 2012) at $d=700 \mathrm{pc}$ (Liseau et al. 1992), and up to $100 \%$ for regions more distant of $1 \mathrm{kpc}$ in the Galactic Plane (Elia et al. 2013). This increasing percentage could be interpreted as a major efficiency in forming gravitationally bound cores in more massive clouds. In the adopted definition of bound cores, however, there 


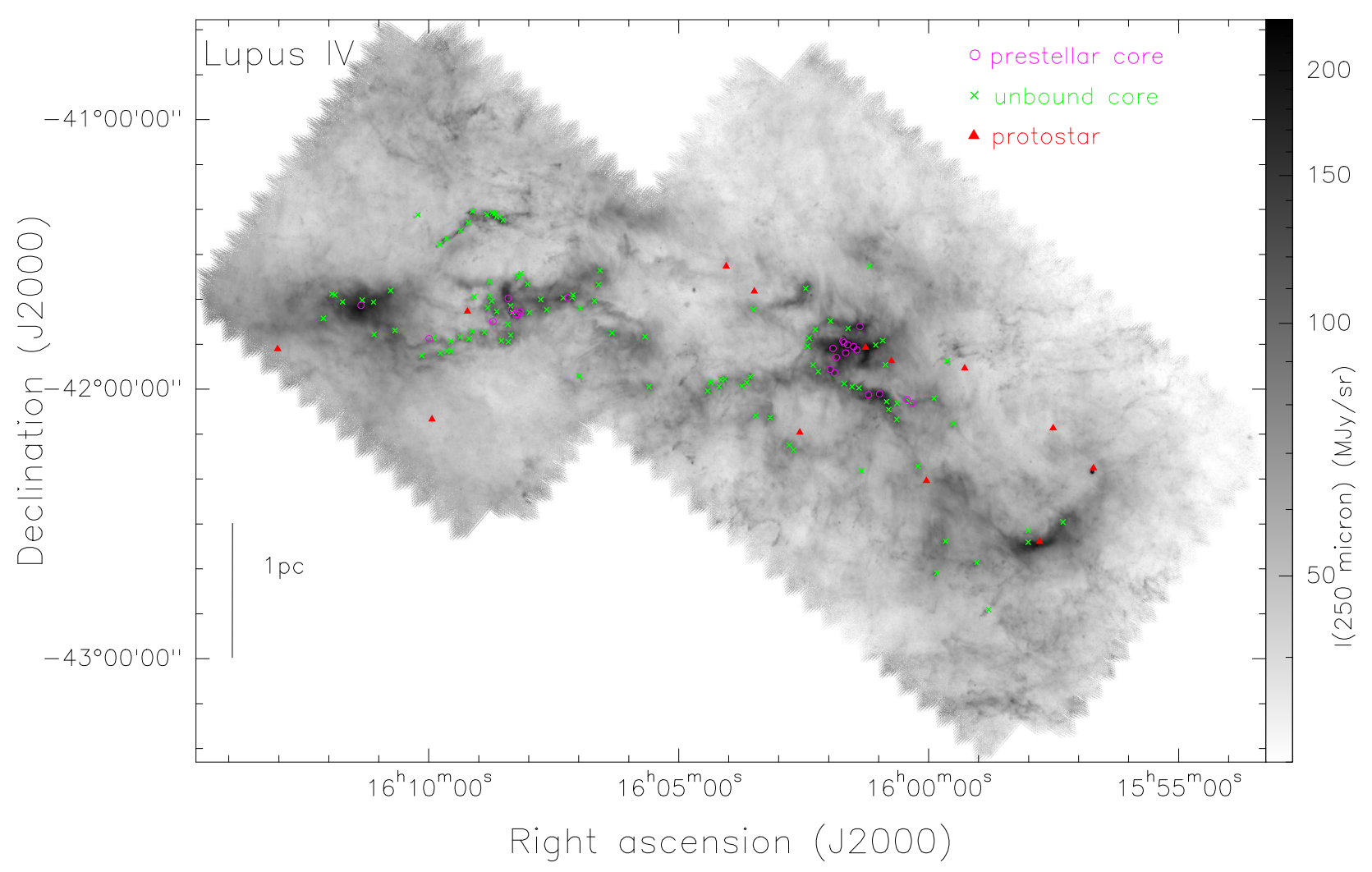

Fig. 3. Herschel $250 \mu \mathrm{m}$ surface brightness image of the Lupus IV region. The selected sample of starless unbound cores (crosses), prestellar cores (circles), and protostars (triangles) are indicated.

is a distance bias since, for a given SED, the derived mass varies as $d^{2}$, while $r_{\mathrm{BE}}$ varies as $d$, thus making it easier for the respective object to fulfil the BE criterion as $d$ increases (Elia et al. 2013). Moreover, there is an observational bias induced by the distance that prevents the detection of smaller and less massive unbound cores in more distant regions. As can be seen looking at the mass versus size plots of the starless cores populations in the mentioned regions (Giannini et al. 2012; Polychroni et al. 2013; Könyves et al. 2015; Marsh et al. 2016), the large number of small $(R \lesssim 0.05 \mathrm{pc})$ and low-mass $\left(M \lesssim 0.01 M_{\odot}\right)$ unbound cores present in Lupus and Taurus are missing in the more distant regions.

\subsection{Completeness of the prestellar core catalogue}

We estimated the completeness of our sample of prestellar cores in the three Lupus clouds by using the following simulation. Briefly, we constructed simulated maps at all Herschel wavelengths (including the column density map) by adding synthetic sources, representative of a genuine population of prestellar cores, to realistic background emission. As background, we used the Herschel images where the emission of the compact sources identified with getsources was subtracted. We then inserted a population of 264 models of BE cores with masses between 0.039 and $0.42 M_{\odot}$, sampling the low-mass end of the observed prestellar core population. The synthetic sources were distributed across the three Lupus clouds to generate a full set of synthetic maps for the five Herschel bands plus the column density image. Compact sources extraction and classification was then performed with getsources for such synthetic skies in the same way as for the observed images. In Fig. 5 we show the completeness curve for the Lupus clouds as a function of the true core

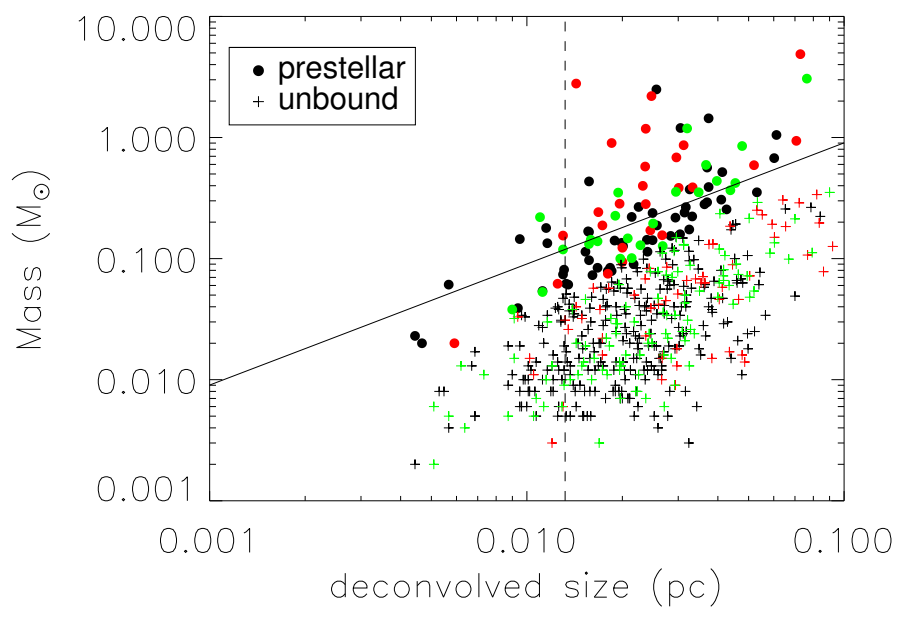

Fig. 4. Mass versus size diagram for the entire population of starless cores. The core sizes were estimated from the geometrical average between the major and minor FWHM measured by getsources in the high-resolution column density map and deconvolved from the $18^{\prime \prime} \cdot 2$ HPBW resolution of the map. The 18 .'2 resolution at the distance of $150 \mathrm{pc}$ is indicated by the dashed line. Candidate prestellar cores are plotted with circular symbols and unbound cores with crosses. Colours indicate cores from the three regions: Lupus I (black), Lupus III (red), Lupus IV (green). The solid line indicates the virial criterion $\left(M_{\mathrm{BE}, \text { crit }} / M_{\mathrm{obs}}=2\right)$, assuming $T=11 \mathrm{~K}$ and $d=150 \mathrm{pc}$; cores above this line are considered robust candidate prestellar cores.

mass. Based on the results of these simulations, we estimate that our Herschel census of candidate prestellar cores is complete at $90 \%$ level above a core mass of $\sim 0.1 M_{\odot}$ (see Fig. 5). A similar completeness level for the Herschel prestellar cores catalogues 


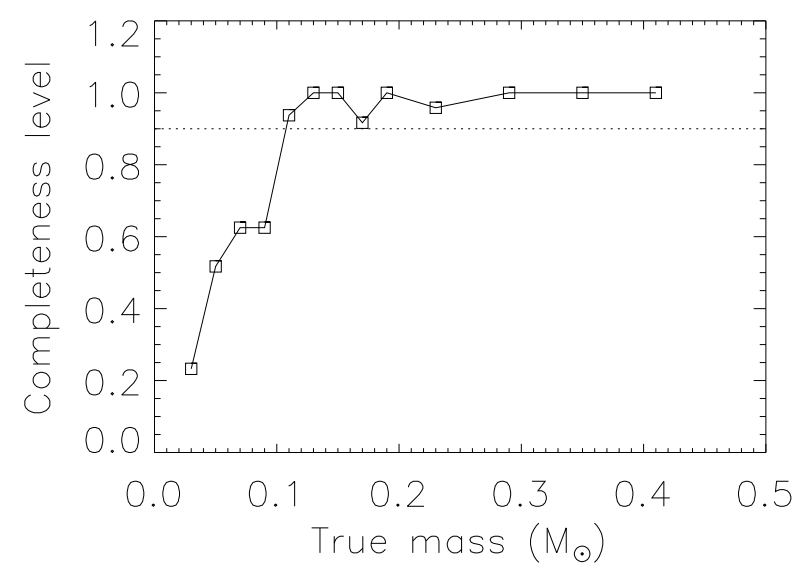

Fig. 5. Completeness level for the synthetic prestellar cores sample added to the Herschel maps as a function of the their mass. From this plot we can estimate that our sample of candidate prestellar cores is complete at $90 \%$ level above a core mass of $\sim 0.1 M_{\odot}$.

was found in Taurus (Marsh et al. 2016) and in Corona Australis (Bresnahan et al. 2018) which are at about the same distance of Lupus.

\section{YSOs/protostars catalogue}

The protostars detected by Herschel in the FIR are objects in different evolutionary stages. They span from extremely young objects - that are still embedded in their parental envelopes and have a strong FIR emission - to more evolved YSOs where the envelopes have been mostly dissipated and the continuum emission is stronger at NIR-MIR wavelengths with only a residual FIR contribution. Therefore, a correct classification of the evolutionary stage of these types of YSOs/protostars requires us to sample the SED as much as possible from NIR to FIR wavelengths. For this purpose, we matched our Herschel catalogue of YSOs and protostars with other infrared catalogues. In particular, we searched for associations within a 6 " radius in the 2Mass, Spitzer, and WISE catalogues and for most of the sources in our catalogue we were able to build a SED spanning from 1.2 to $500 \mu \mathrm{m}$. These SEDs are shown in Fig. 6. In Table A.1 we report the name of the Spitzer and WISE association, if present. We took the 2Mass, Spitzer, and WISE fluxes of the associated sources from the online catalogues by using the Infrared Science Archive (IRSA) $)^{5}$. It is worth noting that in our catalogue we identified some sources (4, 1, and 9 objects in Lupus I, III, and IV, respectively) that are not present in the Spitzer-c2d catalogue of candidates YSOs, because of the wider area covered by Herschel maps. Given the spectral range covered by the Herschel instruments, they are particularly sensitive to protostars in the younger evolutionary stages, namely Class 0 and I, still embedded in a robust dusty envelope responsible for the FIR emission. Consequently, they can miss the most tenuous, evolved YSOs where their envelopes have started to dissipate. Therefore, the Herschel YSOs/protostars catalogue is incomplete for Class II and III objects.

\subsection{SED classification}

The shape of the SED is usually used to classify protostars in evolutionary stages. Four evolutionary classes, Class I, Flat,

\footnotetext{
5 http://irsa.ipac.caltech.edu
}

Class II, and Class III, have been defined by Greene et al. (1994) from the original work of Lada \& Wilking (1984), on the basis of a spectral index $\alpha$, where

$\alpha=\frac{\mathrm{d}\left(\log \left(\lambda F_{\lambda}\right)\right)}{\mathrm{d}(\log \lambda)}$

is the slope, on $\log -\log$ axes, of the SED between 2.2 and $24 \mu \mathrm{m}$. To be consistent with the previous YSOs catalogue of Merin et al. (2008) based on Spitzer data, we used the Spitzer-c2d limits of $\alpha$ for the definition of the four classes (Evans et al. 2009). For each source in our catalogue, we calculated $\alpha$ by using a linear least-squares fit to all available data between $2.2 \mu \mathrm{m}$ ( $K$ band) and $24 \mu \mathrm{m}$ (Spitzer-MIPS). We note that our $\alpha$ index values can differ from those given in the c2d catalogue because we include in the fits both Spitzer and WISE fluxes whenever available. It is worth noting that Herschel measurements can detect also protostars younger than Class I, the so-called Class 0 defined by André et al. (2000) as objects with $L_{\mathrm{smm}} / L_{\mathrm{bol}} \geq 0.01$, where $L_{\mathrm{smm}}$ is the luminosity at $\lambda \geq 350 \mu \mathrm{m}$. We, therefore, evaluated the $L_{\text {smm }}$ of our objects by performing a grey-body fit of the Herschel fluxes at wavelengths $\geq 160 \mu \mathrm{m}$ by using the same opacity law used for dense cores (see Sect. 2) and integrating the emission of the best grey-body fit at $\lambda \geq 350 \mu \mathrm{m}$. Moreover, for all the YSOs/protostars of our Herschel catalogue, we calculated the bolometric luminosity by integrating the observed SED in the widest range of available wavelengths (shown in Fig. 6). $L_{\text {bol }}$, $L_{\mathrm{smm}}, \alpha$ index, and evolutionary class are reported in Table 2.

\subsection{SED fit}

To better define the evolutionary statuses of the YSOs/protostars of our catalogue, we compared their observed SEDs with the set of synthetic SEDs produced by Robitaille (2017). These SEDs are representative of YSOs spanning a wide range of evolutionary stages, from the youngest deeply embedded protostars to pre-main-sequence stars with little or no disk contribution. The full sample of Robitaille (2017) models is divided into 18 sets where the three major elements that determine the SED of a forming star, namely disk, envelope, and cavity, are present or absent and modelled with a different profile. Given the complexity of the model and the large number of input parameters, there is some degeneracy among the synthetic SEDs so that the model that best represents the observed SED is not simply the one with the lowest $\chi^{2}$. In fact, if only one or a small number of models provides a good fit in a specific model set it means that the parameters of such a set need to be fine-tuned to reproduce the data. Hence, that set of models is more unlikely than a different model set where a larger fraction of models can reproduce the data because it requires less fine-tuning. Therefore, instead of simply identifying the best-fit model as the one with the lowest $\chi^{2}$, we identify the best model set as the one with the largest number of good models, following the fitting procedure defined Robitaille (2017) and described in Appendix B.

Given the large numbers of observed fluxes (from 11 to 18 , for most of the sources of our catalogue) and the wide wavelength range, usually only a limited number of model sets are able to reproduce the observed SED reasonably. In Fig. 6, we show the observed SEDs of the $35 \mathrm{YSO}$ /protostar candidates of our catalogue for which the fitting procedure converged, together with the model with the lowest $\chi^{2}$ among the most probable model set. Moreover, in Table 2 we add three columns for three possible input parameters of the SED models: one for the disk, one for the envelope, and one for the cavity. When present, we 
M. Benedettini et al.: A catalogue of dense cores and young stellar objects in the Lupus complex
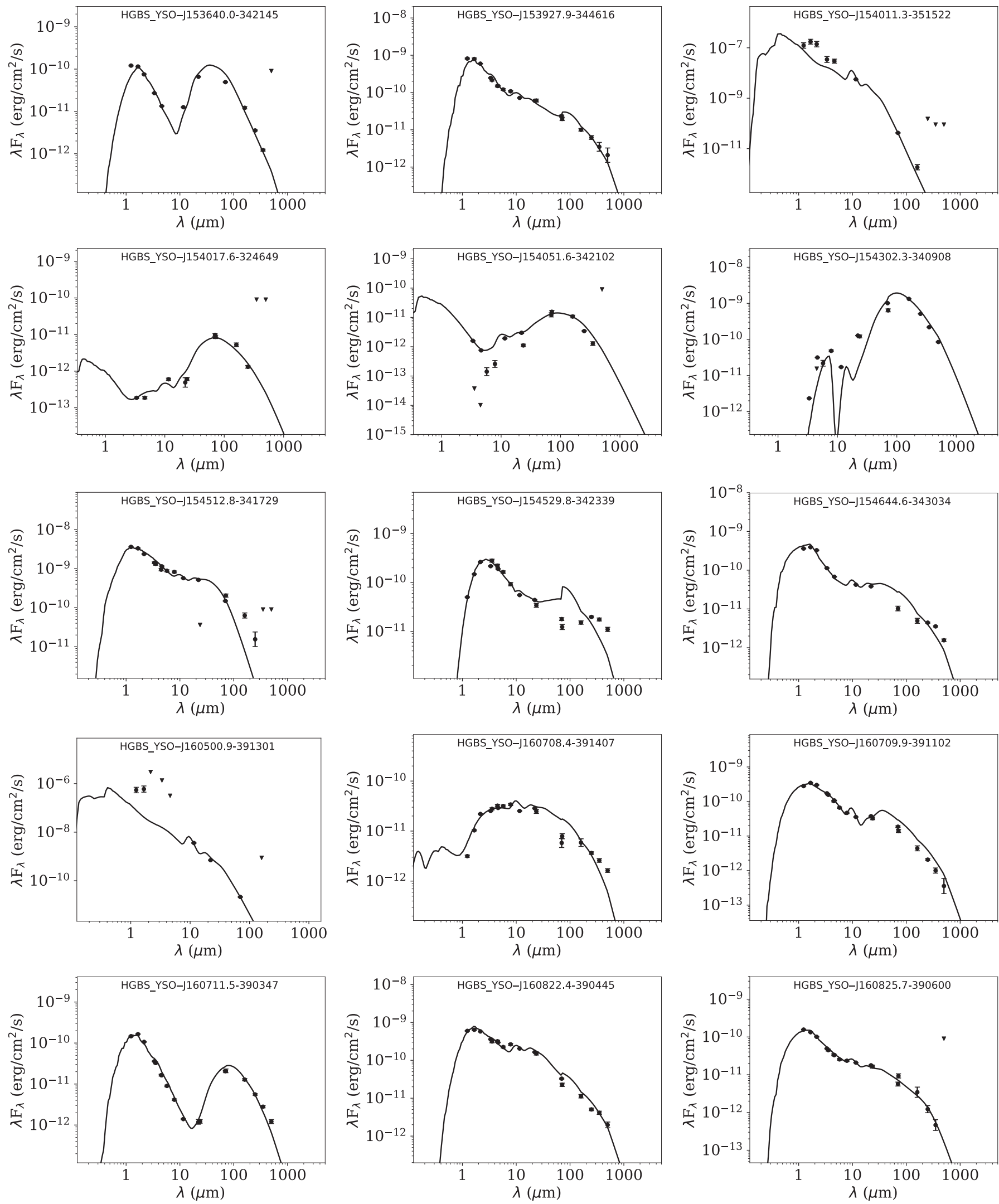

Fig. 6. SED of the YSOs/protostars of the Herschel catalogue from NIR to FIR wavelengths. Triangles are upper limits. The best-fit model of the SED fitting with the Robitaille (2017) synthetic SED is shown.

report the value of the three parameters of the best-fit model. In fact, the presence or absence of these three elements in the best fit model and their relative prominence can give a rough indication of the evolutionary stage of the object. We provide the range of input parameters of all the good models within the best model set (see Appendix B). 

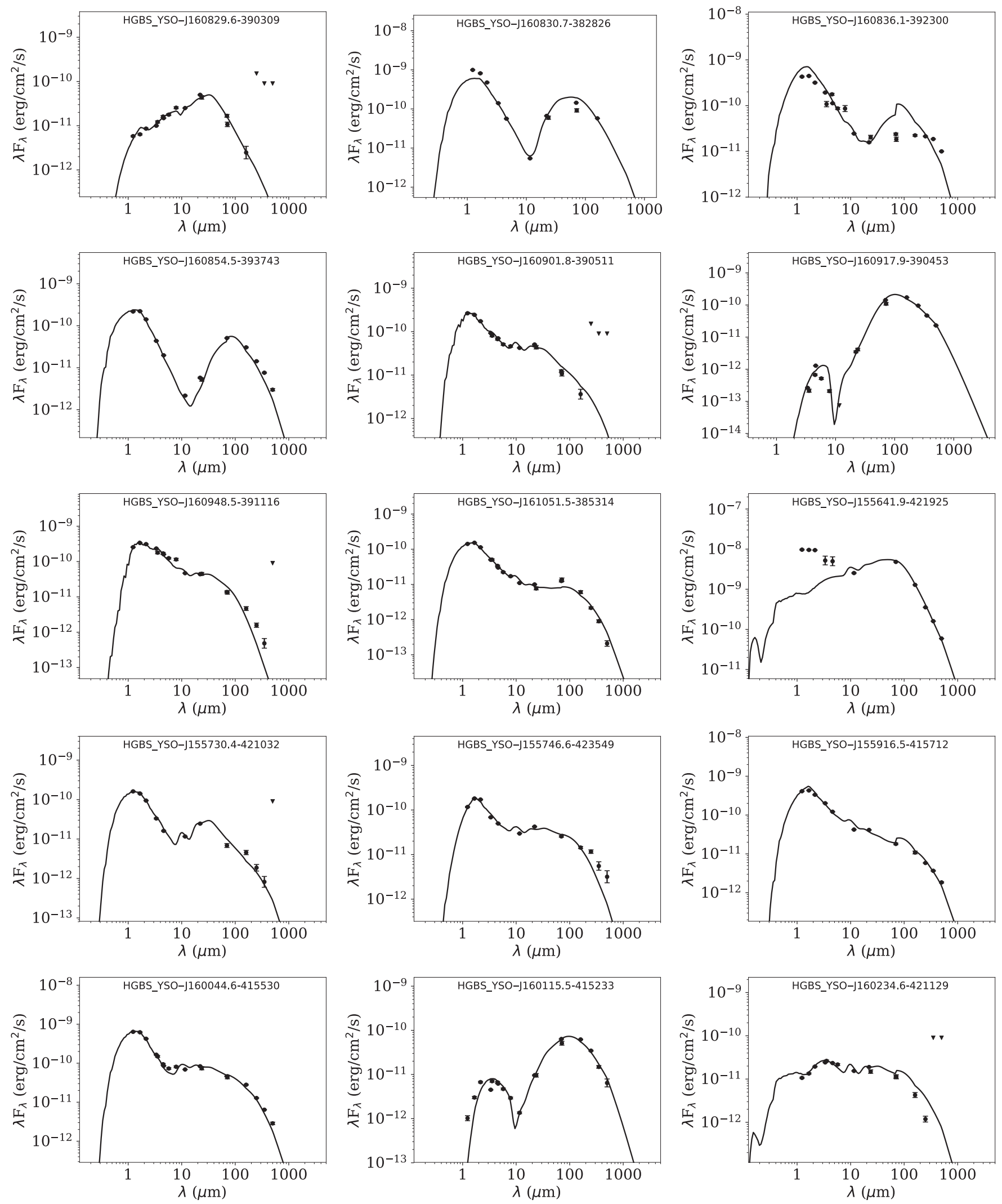

Fig. 6. continued.

\section{Discussion}

\subsection{Comparison with the previous preliminary catalogue}

The total number of detected compact sources (including both starless cores and YSOs/protostars) listed in our catalogue (see
Table 1) is larger than that presented in the preliminary catalogue of Rygl et al. (2013). This increase is not surprising since the Rygl et al. (2013) preliminary catalogue was compiled on maps produced with a different reduction method and using a different source extraction and flux measurement tool (i.e. CuTEx) 

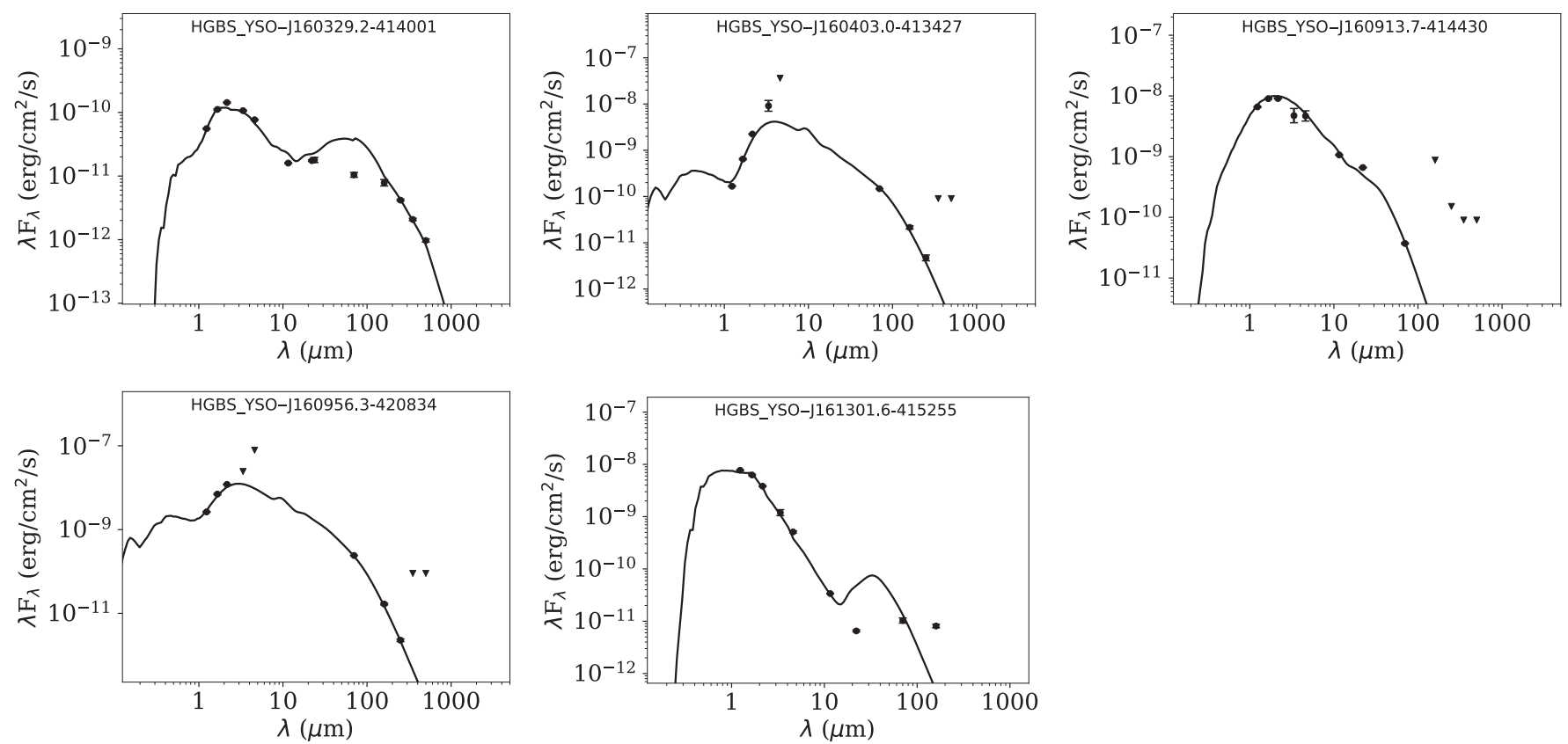

Fig. 6. continued.

and with more stringent criteria for source selection. Hence, differences in the sources identification and classification are expected (see Sect. 3.3). We performed the same analysis of the star formation history done by Rygl et al. (2013) using our more extended catalogue. Specifically, we calculated the ratios of the numbers of observed sources in each evolutionary class (prestellar cores, Class 0, and Class I) with respect to the expected numbers in case of a constant star formation rate (SFR), using the Class II as reference class. We assumed the same lifetimes for each class used by Rygl et al. (2013). In Fig. 7 we show the ratio of observed-to-expected objects numbers for the prestellar cores, Class 0, and Class I objects in each cloud. Despite the difference in absolute numbers with respect to the values in Fig. 3 of Rygl et al. (2013), we find the same trend, confirming their results. For Lupus I we find an increasing SFR with a particularly high ratio for prestellar cores, suggesting that Lupus I is undergoing a major star formation event. Similarly, for Lupus IV the observed number of prestellar cores and Class 0 is higher than what expected for a constant SFR, suggesting that the star formation has accelerated over the past $\sim 0.5$ Myr. Conversely, in Lupus III the bulk of the star formation activity has already passed and has decelerated in the last $\sim 2 \mathrm{Myr}$, with a recent small increment of the prestellar cores formation.

\subsection{Spatial distribution of sources and cloud clumpiness}

From Figs. 1-3, we see that the different categories of objects identified by Herschel, namely unbound starless cores, prestellar cores, and YSOs/protostars, are differently distributed across the regions, with the prestellar cores clustered in spatially limited parts of the clouds, corresponding to the brighter and higher column density filamentary regions, while the majority of the lower column density filaments are populated by unbound starless cores. Conversely, YSOs are spread across the full cloud, even in regions with very low column density and they cannot be associated with any filament. To quantify this visual impression, we calculated the percentages of association with filaments for each of the three categories. We used the filaments identification

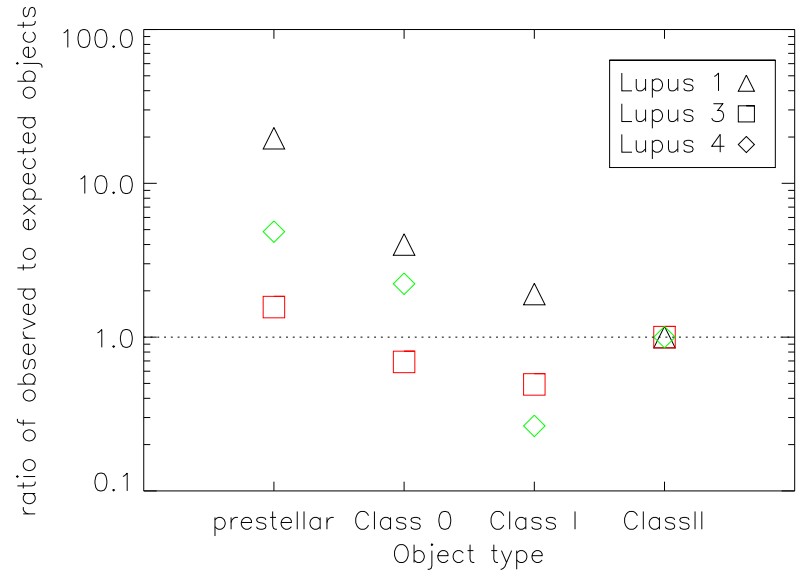

Fig. 7. Ratio of observed objects number for each evolutionary class with respect to the expected number for a constant SFR. Values above 1 indicate an accelerating SFR. Values below 1 indicate a decelerating SFR. Different symbols and colours are used for the ratios of the three Lupus clouds, as in the legend.

presented in Paper I and considered that a source is associated with a filament if its central position falls within the boundary of the filament. We found different percentages of association for the three type of sources. Only $26 \%$ of YSOs/protostars and the $36 \%$ of the unbound cores are associated with filaments while almost all (94\%) prestellar cores are within a filament, in particular close to its central, brightest crest. High percentages of association with filaments for the prestellar cores are found also in other star-forming regions within the HGBS project, showing that filaments are the preferred place where the dense condensations - that are the seeds of new stars - are formed (André et al. 2014 and references therein). On the other hand, YSOs have a lower probability to be associated with filaments because this type of objects during their evolution from the prestellar phase to the YSO phase have had time to migrate from their original position and/or the filament where they were formed is dissipated. 
Table 2. SED classification of the YSOs/protostars in our catalogue.

\begin{tabular}{|c|c|c|c|c|c|c|c|c|c|}
\hline $\begin{array}{c}\text { Name } \\
\text { HGBS_YSO_- }\end{array}$ & $\begin{array}{c}\text { RA (J2000) } \\
(\text { h m s })\end{array}$ & $\begin{array}{c}\operatorname{Dec}(\mathrm{J} 200) \\
\left({ }^{\circ},{ }^{\prime \prime}\right) \\
\end{array}$ & $\begin{array}{l}L_{\text {bol }} \\
\left(L_{\odot}\right) \\
\end{array}$ & $\begin{array}{c}L_{\mathrm{smm}} \\
\left(L_{\odot}\right) \\
\end{array}$ & $\alpha$ & Class & $\begin{array}{c}M_{\text {disk }}{ }^{a} \\
\left(M_{\odot}\right)\end{array}$ & $\begin{array}{c}\rho_{0}^{\mathrm{env} b} \\
\left(\mathrm{~g} \mathrm{~cm}^{-3}\right) \\
\end{array}$ & $\begin{array}{c}\rho_{0}^{\text {cav } c} \\
\left(\mathrm{~g} \mathrm{~cm}^{-3}\right) \\
\end{array}$ \\
\hline \multicolumn{10}{|l|}{ LUPUS I } \\
\hline J153640.0-342145 & 153640.0 & -342146.6 & $6.8 \mathrm{e}-01$ & $1.7 \mathrm{e}-04$ & -0.071 & Flat & $3.052 \mathrm{e}-04$ & $1.377 \mathrm{e}-21$ & $2.442 \mathrm{e}-21$ \\
\hline $\mathrm{J} 153927.9-344616$ & 153928.0 & -344617.8 & $3.5 \mathrm{e}+00$ & $9.1 \mathrm{e}-04$ & -0.826 & II & $8.815 \mathrm{e}-04$ & $4.996 \mathrm{e}-23$ & $1.159 \mathrm{e}-23$ \\
\hline $\mathrm{J} 154011.3-351522$ & 154011.3 & $\begin{array}{lll}-35 & 15 & 22.4\end{array}$ & $3.7 e+02$ & $0.0 \mathrm{e}+00$ & -1.820 & III & $3.318 \mathrm{e}-06$ & & (1) \\
\hline $\mathrm{J} 154017.6-324649$ & 154017.7 & -324649.1 & $4.7 \mathrm{e}-03$ & $5.6 e-05$ & 0.649 & $\mathrm{I} / 0$ & $2.754 \mathrm{e}-07$ & $2.765 \mathrm{e}-19$ & $1.813 \mathrm{e}-21$ \\
\hline J154051.6-342102 & 154051.6 & -342103.7 & $2.1 \mathrm{e}-02$ & $1.9 \mathrm{e}-04$ & 0.587 & I & $2.137 \mathrm{e}-02$ & $8.265 \mathrm{e}-22$ & $7.478 \mathrm{e}-23$ \\
\hline $\mathrm{J} 154302.3-340908$ & 154302.4 & -340908.2 & $2.9 \mathrm{e}-01$ & $3.5 \mathrm{e}-02$ & 1.469 & 0 & & $3.017 \mathrm{e}-18$ & $1.519 \mathrm{e}-23$ \\
\hline $\mathrm{J} 154512.8-341729$ & 154512.9 & -341730.8 & $1.1 \mathrm{e}+01$ & $7.5 e-04$ & -0.637 & II & $7.914 \mathrm{e}-05$ & & \\
\hline $\mathrm{J} 154529.8-342339$ & 154529.9 & -342339.2 & $2.3 e-01$ & $5.7 \mathrm{e}-03$ & -0.952 & $\mathrm{II} / 0$ & $9.364 \mathrm{e}-02$ & $1.244 \mathrm{e}-23$ & $3.096 \mathrm{e}-23$ \\
\hline $\mathrm{J} 154644.6-343034$ & 154644.7 & -343035.9 & $2.6 e+00$ & $7.3 e-04$ & -0.835 & II & $5.221 \mathrm{e}-05$ & $3.589 \mathrm{e}-24$ & $4.890 \mathrm{e}-22$ \\
\hline \multicolumn{10}{|l|}{ LUPUS III } \\
\hline J160500.9-391301 & 160501.0 & -391301.2 & $1.0 \mathrm{e}+03$ & $0.0 \mathrm{e}+00$ & -2.625 & III & $5.243 \mathrm{e}-06$ & & \\
\hline $\mathrm{J} 160708.4-391407$ & 160708.4 & -391408.7 & $7.4 \mathrm{e}-02$ & $9.1 \mathrm{e}-04$ & 0.018 & Flat/0 & $7.356 \mathrm{e}-05$ & $9.630 \mathrm{e}-24$ & \\
\hline $\mathrm{J} 160709.9-391102$ & 160710.0 & -391103.9 & $2.1 \mathrm{e}+00$ & $3.2 \mathrm{e}-04$ & -0.894 & II & $2.619 \mathrm{e}-08$ & $6.397 \mathrm{e}-20$ & $2.907 \mathrm{e}-21$ \\
\hline J160711.5-390347 & 160711.5 & -390347.3 & $1.1 \mathrm{e}+00$ & $7.8 \mathrm{e}-04$ & -1.910 & III & $1.406 \mathrm{e}-08$ & $1.208 \mathrm{e}-21$ & $2.167 \mathrm{e}-23$ \\
\hline $\mathrm{J} 160822.4-390445$ & 160822.4 & -390445.2 & $4.6 e+00$ & $1.2 \mathrm{e}-03$ & -0.464 & II & $1.017 \mathrm{e}-03$ & $4.002 \mathrm{e}-22$ & $4.129 \mathrm{e}-22$ \\
\hline $\mathrm{J} 160825.7-390600$ & 160825.7 & -390601.7 & $1.1 \mathrm{e}+00$ & $1.3 e-04$ & -0.627 & II & $5.053 \mathrm{e}-03$ & & \\
\hline J160829.6-390309 & 160829.6 & -390310.8 & $1.4 \mathrm{e}-02$ & $0.0 \mathrm{e}+00$ & 0.721 & $\mathrm{I}$ & $\ldots$ & $1.769 \mathrm{e}-21$ & $1.358 \mathrm{e}-22$ \\
\hline $\mathrm{J} 160830.7-382826$ & 160830.7 & -382826.5 & $3.8 \mathrm{e}+00$ & $0.0 \mathrm{e}+00$ & -0.832 & II & $1.878 \mathrm{e}-02$ & $1.952 \mathrm{e}-23$ & $1.538 \mathrm{e}-22$ \\
\hline J160834.1-390617 & 160834.2 & -390617.4 & $1.8 \mathrm{e}+02$ & $4.6 e-04$ & -1.463 & II & no fit & & \\
\hline $\mathrm{J} 160836.1-392300$ & 160836.2 & -392301.9 & $3.3 e+00$ & $7.7 \mathrm{e}-03$ & -1.225 & II & $6.117 \mathrm{e}-02$ & $3.771 \mathrm{e}-23$ & $9.300 \mathrm{e}-23$ \\
\hline $\mathrm{J} 160854.5-393743$ & 160854.6 & -393743.5 & $2.8 \mathrm{e}+00$ & $2.1 \mathrm{e}-03$ & -1.352 & II & $5.094 \mathrm{e}-02$ & & \\
\hline $\mathrm{J} 160901.8-390511$ & 160901.8 & -390511.2 & $8.5 e-01$ & $0.0 \mathrm{e}+00$ & -0.447 & II & $6.486 \mathrm{e}-04$ & $1.128 \mathrm{e}-23$ & $5.924 \mathrm{e}-22$ \\
\hline J160917.9-390453 & 160917.9 & -390453.0 & $9.9 \mathrm{e}-02$ & $1.5 \mathrm{e}-02$ & 1.235 & 0 & $4.764 \mathrm{e}-06$ & $1.235 \mathrm{e}-19$ & $1.081 \mathrm{e}-22$ \\
\hline J160948.5-391116 & 160948.5 & $-3911 \quad 16.4$ & $1.8 \mathrm{e}+00$ & $1.5 \mathrm{e}-04$ & -0.863 & II & $1.506 \mathrm{e}-04$ & & \\
\hline $\mathrm{J} 161051.5-385314$ & 161051.5 & -385314.1 & $1.1 \mathrm{e}+00$ & $2.2 \mathrm{e}-04$ & -1.015 & II & $2.380 \mathrm{e}-03$ & $3.772 \mathrm{e}-23$ & $2.516 \mathrm{e}-23$ \\
\hline J161134.4-390008 & 161134.4 & -390008.2 & $3.9 \mathrm{e}+01$ & $3.6 e-04$ & -1.572 & II & no fit & & \\
\hline \multicolumn{10}{|l|}{ LUPUS IV } \\
\hline J155641.9-421925 & 155642.0 & -421925.3 & $2.6 e+01$ & $1.9 \mathrm{e}-02$ & -0.749 & II & $8.610 \mathrm{e}-03$ & $2.970 \mathrm{e}-19$ & $1.009 \mathrm{e}-23$ \\
\hline $\mathrm{J} 155730.4-421032$ & 155730.5 & $\begin{array}{llll}-42 & 10 & 32.3\end{array}$ & $8.1 \mathrm{e}-01$ & $1.3 e-04$ & -0.549 & II & 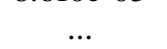 & $3.093 \mathrm{e}-20$ & $\ldots$ \\
\hline $\mathrm{J} 155746.6-423549$ & 155746.6 & -423550.8 & $9.4 \mathrm{e}-01$ & $1.4 \mathrm{e}-03$ & -0.569 & II & $6.572 \mathrm{e}-03$ & $4.594 \mathrm{e}-24$ & $12.164 \mathrm{e}-22$ \\
\hline J155916.5-415712 & 155916.6 & -415712.2 & $2.6 e+00$ & $6.5 e-04$ & -0.957 & II & $1.687 \mathrm{e}-02$ & (2) & $\ldots$ \\
\hline $\mathrm{J} 160002.5-422216$ & 160002.5 & -422216.3 & $7.2 \mathrm{e}-01$ & $4.8 \mathrm{e}-04$ & -1.124 & II & no fit & & \\
\hline $\mathrm{J} 160044.6-415530$ & 160044.6 & -415531.9 & $2.8 \mathrm{e}+00$ & $1.1 \mathrm{e}-03$ & -0.502 & II & $1.368 \mathrm{e}-02$ & $3.488 \mathrm{e}-24$ & $3.113 \mathrm{e}-21$ \\
\hline $\mathrm{J} 160115.5-415233$ & 160115.6 & -415234.9 & $3.7 \mathrm{e}-02$ & $3.0 \mathrm{e}-03$ & 0.015 & Flat/0 & $4.258 \mathrm{e}-08$ & $1.126 \mathrm{e}-20$ & $2.154 \mathrm{e}-23$ \\
\hline $\mathrm{J} 160234.6-421129$ & 160234.6 & $\begin{array}{lll}-42 & 11 & 29.3\end{array}$ & $3.8 \mathrm{e}-02$ & $5.4 \mathrm{e}-05$ & -0.164 & Flat & $1.009 \mathrm{e}-03$ & $1.153 e-24$ & $1.197 \mathrm{e}-22$ \\
\hline $\mathrm{J} 160329.2-414001$ & 160329.2 & -414001.2 & $4.1 \mathrm{e}-01$ & $3.5 e-04$ & -0.965 & II & $3.946 \mathrm{e}-04$ & $1.768 \mathrm{e}-24$ & $7.543 e-21$ \\
\hline J160403.0-413427 & 160403.0 & -413427.1 & $3.4 \mathrm{e}+02$ & $0.0 \mathrm{e}+00$ & $\ldots$ & $\ldots$ & $6.677 \mathrm{e}-04$ & $\ldots$ & $\ldots$ \\
\hline $\mathrm{J} 160913.7-414430$ & 160913.7 & -414430.5 & $2.5 e+01$ & $0.0 \mathrm{e}+00$ & -1.170 & II & $1.031 \mathrm{e}-05$ & $\ldots$ & $\ldots$ \\
\hline $\mathrm{J} 160956.3-420834$ & 160956.3 & -420834.2 & $5.7 e+02$ & $0.0 \mathrm{e}+00$ & $\ldots$ & $\ldots$ & $1.218 \mathrm{e}-04$ & $\ldots$ & $\ldots$ \\
\hline J161301.6-415255 & 161301.7 & -415255.5 & $2.8 \mathrm{e}+01$ & $0.0 \mathrm{e}+00$ & -2.772 & III & $5.518 \mathrm{e}-06$ & $\ldots$ & $\ldots$ \\
\hline
\end{tabular}

Notes. Three parameters of the SED fit, disk mass $\left(M_{\text {disk }}\right)$, envelope density at the reference radius $\left(\rho_{0}^{\text {env }}\right)$, and cavity density at the reference radius $\left(\rho_{0}^{\text {cav }}\right)$, are listed to indicate the presence of a disk, envelope and cavity in the best-fit model of the observed SED, respectively. Objects for which the SED fit fails are indicated with "no fit" in the eighth column. ${ }^{(a)} M_{\text {disk }}$ is the mass of the disk. ${ }^{(b)} \rho_{0}^{\text {env }}$ is the envelope density at the reference radius $r_{0}$ and it is serves to scale the envelope density. ${ }^{(c)} \rho_{0}^{\text {cav }}$ is the constant density in the inner regions of the cavity where $\rho_{0}^{\text {cav }}$ is lower than the envelope density; in the other regions, the cavity density is set to be equal to the envelope density.

We found that about one third of YSOs/protostars and unbound cores (26\% and $36 \%$, respectively) are associated with filaments, while almost all $(94 \%)$ prestellar cores are associated with filaments. This behaviour is in line with one of the main results of the Herschel photometric surveys of star-forming regions that filaments are the preferred place where the dense condensations - that are the seeds of new stars - are formed (André et al. 2014 and references therein). The locations of the different types of objects also show that star formation activity is not uniformly distributed across the clouds. As examples, in Lupus I the north-west part of the cloud is populated by only starless cores; in Lupus III the majority of prestellar cores are in the long brightest filament crossing the cloud east-west; and in Lupus IV the prestellar cores are clustered in two locations around RA of $16^{\mathrm{h}} 01^{\mathrm{m}} 00^{\mathrm{s}}$ and $16^{\mathrm{h}} 08^{\mathrm{m}} 00^{\mathrm{s}}$.

The total number of starless cores (unbound plus prestellar) in Lupus $\mathrm{I}$ is about three times that of the other two clouds, suggesting a possible difference in the level of clumpiness of 
the three Lupus clouds. To estimate this level, we calculated the number of starless cores per $\mathrm{pc}^{2}$ above a certain column density level for the three regions. We choose a column density contour of $1.8 \times 10^{21} \mathrm{~cm}^{-2}$, corresponding to a visual extinction of $2 \mathrm{mag}$, since it roughly corresponds to the border of the filamentary clouds. Within this contour, we obtained 100 starless cores per $\mathrm{pc}^{2}$ in Lupus I, 29 starless cores per $\mathrm{pc}^{2}$ in Lupus III, and 52 starless cores per $\mathrm{pc}^{2}$ in Lupus IV. Therefore, in Lupus I the number of starless cores per $\mathrm{pc}^{2}$ is a factor of 2 higher than in Lupus IV and a factor 3.4 higher than in Lupus III, confirming a higher level of clumpiness in the Lupus I cloud. To investigate this aspect more deeply, we calculated another estimator of the level of clumpiness, namely the projected distance from the nearest neighbour. For the starless cores sample, we found that the nearest-neighbour distance in Lupus I has the lowest median value $(0.07 \mathrm{pc})$, it has a slightly higher value $(0.08 \mathrm{pc})$ in Lupus IV, while in Lupus III we found the highest value $(0.1 \mathrm{pc})$. We then used the Kolmogoroff-Smirnov (K-S) test to compare the distributions of the nearest-neighbour distance in the three clouds to see at what level they are different. With this test, we found that it is highly improbable that the nearest-neighbour distances of starless cores in Lupus I and III belong to the same kind of distribution (K-S probability $p=0.0002)$. Similarly, we found very low $\mathrm{K}-\mathrm{S}$ probabilities when we compare the nearestneighbour distributions of Lupus I and Lupus IV $(p=0.01)$ and Lupus III and Lupus IV $(p=0.13)$.

All the previous evidence indicates that Lupus $\mathrm{I}$ is the cloud with the highest level of clumpiness. Since the Lupus sub regions - in general - appear quite similar, we wonder what is the origin of Lupus I's higher level of clumpiness. One possibility could be that it is at a younger evolutionary stage in terms of star formation activity (Hughes et al. 1994; Benedettini et al. 2012; Rygl et al. 2013). Indeed, a lower number of starless cores should be a natural effect of the evolution of the star formation process. In the absence of continuous infall of gas available for forming new dense cores, the number of dense cores should decrease over a typical time related to the core lifetime, due to the core dissipation or evolution into protostars. The typical lifetime of the Herschel starless cores estimated in the Aquila region is $\sim 1 \mathrm{Myr}$ (Könyves et al. 2015). In Lupus I, star formation activity at the present epoch is proceeding at an increasing rate and the ages of the stellar population are usually lower than $1 \mathrm{Myr}$ (Hughes et al. 1994). Therefore, the dense cores created since the onset of the current star formation activity should be, on average, still present. In contrast, in Lupus III we measured a decreasing SFR rate at the present epoch, indicating that the bulk of the star formation activity has passed. On average, the stars in Lupus III are older than 4 Myr (Mortier et al. 2011), therefore they have an age greater than the fiducial Herschel prestellar cores lifetime ( $\sim 1$ Myr), suggesting that most of the cores formed at the onset of past major star formation events have already been vanished.

Another possibility is that the higher level of clumpiness of Lupus I is a side effect of the collision of the flows from which it was generated. Indeed, much evidence has been collected (Tothill et al. 2009; Paper I; Gaczkowski et al. 2017) showing that Lupus I could have been strongly influenced by colliding flows generated by the expanding Upper-Scorpius H I shell and the Upper Centaurus-Lupus wind bubble. Furthermore, theoretical models have shown that shocked flows are a very efficient mechanism for the formation of filaments and the density perturbations that generate dense cores in molecular clouds (e.g. Padoan et al. 2001; Heitsch \& Hartmann 2008; Inutsuka et al. 2015). Conversely, the cloud material in Lupus III and IV, even though they belong to the same molecular complex as Lupus I,

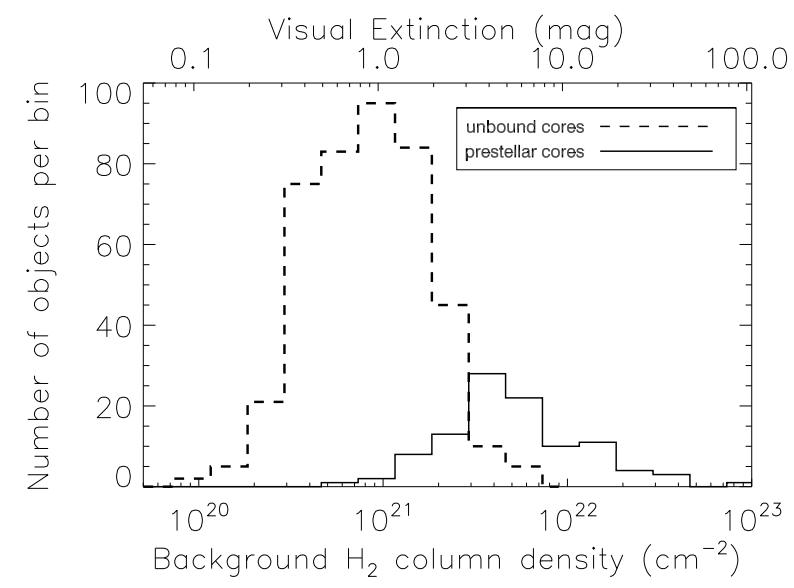

Fig. 8. Histogram of the background column density for the sample of unbound cores (dashed line) and prestellar cores (solid line).

have not been affected by the expanding shells, and so have not yet experienced any major collision.

\subsection{About the existence of a column density threshold for the formation of prestellar cores}

In Fig. 8, we show the histograms of unbound cores and prestellar cores as function of their local background $\mathrm{H}_{2}$ column density. The distributions of the two categories are well separated. Unbound starless cores are found over lower column density backgrounds (from $\sim 10^{20}$ to $\sim 6 \times 10^{21} \mathrm{~cm}^{-2}$ ), while prestellar cores are found above a background of $N\left(\mathrm{H}_{2}\right)>8 \times 10^{20} \mathrm{~cm}^{-2}$ with a modal value corresponding to $\sim 4$ mag of visual extinction, assuming the Bohlin et al. (1978) conversion factor $N\left(\mathrm{H}_{2}\right) / A_{\mathrm{V}}=$ $9.4 \times 10^{20}\left(\mathrm{~cm}^{-2} \mathrm{mag}^{-1}\right)$.

The fragmentation of filaments in roundish cores is a key step of the passage of material from clouds to stars, yet it seems that only cores above a certain background $\mathrm{H}_{2}$ column density threshold become gravitationally bound and eventually evolve into protostars. Theoretically, a column density threshold is expected since in low density environments material is not well shielded from the interstellar radiation field and correspondingly its ionisation fraction is high. In this case, the magnetic pressure becomes efficient in opposing gravitational collapse and limiting the formation of substructures (McKee 1989). In the last few decades, some observational evidence for the existence of an $\mathrm{H}_{2}$ column density threshold for star formation has been collected from millimetre and submillimetre surveys of dense cores correlated to NIR extinction maps (Onishi et al. 1998; Johnstone et al. 2004; Kirk et al. 2006). These surveys, however, suffer from severe limitations, including their limited capability in probing the full population of dense cores, their limited dynamical range, and the different tracers used to detect the cores and estimate the visual extinction. In this respect, the Herschel data represent a step forward since they have the advantages of being able to probe prestellar cores and their underlying background simultaneously and of being more sensitive to the detection of dense cores, reaching the level of completeness for core masses above $\sim 0.1 M_{\odot}$ (Könyves et al. 2015; Marsh et al. 2016; Bresnahan et al. 2018).

In earlier analyses of Herschel catalogues, the visual extinction threshold for star formation was defined as the background level above which $90 \%$ of prestellar cores are found. Values of about 7 and 6 mag were found for Aquila and Taurus, respectively (Könyves et al. 2015; Marsh et al. 2016). Ground-based 
estimates of the threshold are available only for a handful of regions and a large range of values were found for different regions, for example, from $\sim 5$ mag in Perseus (Kirk et al. 2006) to $\sim 9$ mag in Taurus (Onishi et al. 1998). In Lupus, we find a threshold of $\sim 2 \mathrm{mag}$, meaning that $90 \%$ of prestellar cores lie on a background higher than this threshold. Even considering the large uncertainties affecting our $\mathrm{H}_{2}$ column densities, which could be underestimated by up to factor of 2 , the visual extinction threshold found in Lupus is lower than those measured in other star-forming clouds so far.

It is worth noting that Lupus is a peculiar star-forming cloud complex in term of mass density. For example, it is characterised by very low column densities both for its diffuse medium and for its denser material assembled in filaments. More specifically, the probability distribution function of column density (PDF) in Lupus has a peak equivalent to a visual extinction of less than $1 \mathrm{mag}$, the lowest value found so far in PDF analyses of star-forming clouds based on Herschel data, that usually peak at visual extinctions larger than $1.5 \mathrm{mag}$ (Paper I). Moreover, the Lupus filaments have average column densities at the lower end of the distribution of filaments column densities found in other clouds (Paper I; Arzoumanian et al. 2013). Despite these lower column densities, star formation is ongoing in the Lupus I, III, and IV clouds and we find robust candidate prestellar cores even on backgrounds levels lower than what is usually found in other clouds. The presence of dense cores, some of which have been considered prestellar, in cloud regions of $A_{\mathrm{v}} \lesssim 5$ mag has been observed not only in Lupus but also in Perseus (Hatchell et al. 2005) and Taurus (Marsh et al. 2016). Therefore, the column density threshold should be interpreted more as a level over which a higher probability exists to find prestellar cores rather than a stringent limit under which star formation is inhibited (Hatchell et al. 2005; Lada et al. 2010; André et al. 2014; Könyves et al. 2015). Moreover, the fact that different values of the threshold are found in different star-forming clouds is an indication that the star formation column density threshold, if it exists, might depend on the local properties of the host cloud such as the strength of its magnetic field, the local radiation field, and its non-thermal velocity dispersion. In fact, these properties control the mechanisms that can provide support against gravitational collapse, such as the magnetic pressure mediated by collisions between neutrals and ions and the turbulent motions that supply non-thermal pressure support.

This conclusion is also supported by the results of an alternative method to look for the possible presence of a star formation threshold in GMCs that is to investigate the relation between the surface densities of the SFR and gas mass, namely the Schmidt conjecture. By applying the Bayesian method developed by Lombardi et al. (2013) to four GMCs, Lada et al. (2013) studied the power-law relation between the protostellar surface density distribution derived from Spitzer catalogues of protostars and the dust surface density distribution measured by the extinction maps derived from 2MASS data. They found that there is no star formation threshold for two clouds, Orion A and Taurus, while for the other two clouds, Orion B and California, data are compatible with the possible presence of a threshold, even if with a large degree of uncertainty.

\subsection{Prestellar cores associated with filaments on average thermally subcritical}

In Paper I, we highlighted that the majority of filaments found in Lupus have a mass per unit length lower than the maximum value needed to be thermally supported. This critical value for

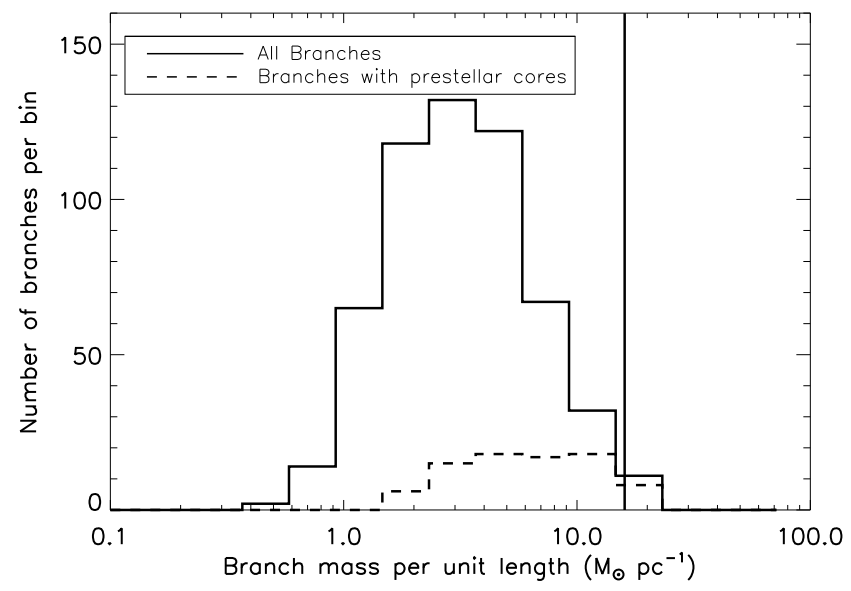

Fig. 9. Distribution of the average mass per unit length of the filament branches (solid line). Dashed line represents the subset of filament branches associated with prestellar cores. The vertical bar indicates the critical value of $16 M_{\odot} \mathrm{pc}^{-1}$ for radial gravitational collapse of a cylindrical, isothermal filament with a temperature of $10 \mathrm{~K}$.

isothermal infinite cylindrical filaments with a typical temperature of $10 \mathrm{~K}$, confined by the external pressure of the ambient medium, is $\sim 16 M_{\odot} \mathrm{pc}^{-1}$ (see Fig. 9). Previous studies (André et al. 2010, 2014; Arzoumanian et al. 2011) have suggested that only filaments with masses per unit length above this critical value are primarily associated with star formation activity. In Lupus, however, there is evident star formation activity even though the large majority of filaments are subcritical. More specifically, we find that subcritical filaments contain prestellar core candidates in which new stars may form (see Fig. 9).

We stress that this finding is still valid even if we consider the large uncertainty associated with the mass estimates of both filaments and cores. In Paper I we estimated the mass per unit length of a filament by assuming that the total mass measured in the structure was uniformly distributed along the entire structure, meaning that we give the average value along the filament. On the other hand, other studies use a different measure to estimate mass per unit length, considering the average $\mathrm{H}_{2}$ column density of only the denser central part of the filament. The two values differ by a factor of about 1.5 , well below the uncertainty associated with the filament column density (Schisano et al. 2014; Paper I).

In Lupus, the fact that prestellar cores are observed in some of its supposedly globally subcritical filaments is a indication that the condition of overdensity needed to activate gravitational collapse can be reached only locally and is not necessarily a global property of the filament. Indeed, filaments are highly irregular structures with significant fluctuations of brightness and column density along the principal spine (Schisano et al. 2014; Paper I). We conclude that in a low column density regime, such as that of the Lupus clouds, the average mass per unit length is not a good parameter for identifying those filaments where star formation primarily takes place, since a significant fraction of stars may form along filaments that are globally subcritical.

\subsection{Core mass function}

In Fig. 10, we show histograms of the temperatures and masses of the prestellar cores sample for the three Lupus clouds. The temperature distribution is very similar in the three clouds, ranging between 7 and $13 \mathrm{~K}$ with a median value around $10 \mathrm{~K}$. 

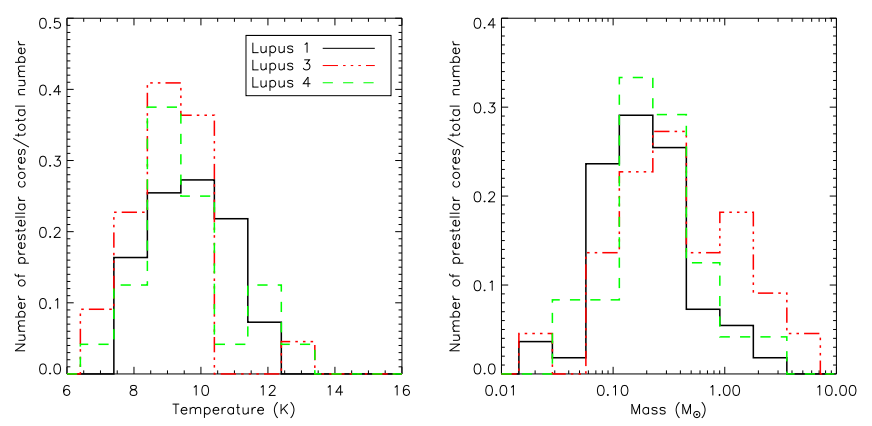

Fig. 10. Normalised distribution of the temperature (left panel) and mass (right panel) of the population of prestellar cores in the three Lupus clouds. On the $y$-axis, the number of objects per bin relative to the total number of prestellar cores in each cloud is reported.

These values are typical values for the prestellar cores identified from Herschel data of both low- and high-mass star-forming regions (e.g. Giannini et al. 2012; Könyves et al. 2015; Marsh et al. 2016; Rayner et al. 2017). Temperatures around $10 \mathrm{~K}$ are also the typical values for gas kinetic temperature and dust temperature derived in starless dense cores from single-dish submillimetre observations (e.g. Tafalla et al. 2002; Foster et al. 2009; Keown et al. 2017). As noted earlier in Sect. 4.1, however, the temperature derived from SED fitting of Herschel data is an averaged temperature. Indeed, interferometric observations have shown that dense starless cores are not isothermal and have temperatures that drop below $10 \mathrm{~K}$ at the core centre (e.g. Tafalla et al. 2004; Crapsi et al. 2007). Furthermore, in the outer regions of cores, where density and shielding from the external radiation field are smaller, the gas and dust temperatures become uncoupled (Galli et al. 2002).

The mass distributions of prestellar cores span a similar range for the three Lupus clouds, with median values of the core masses in the three clouds of $0.17,0.38$, and $0.22 M_{\odot}$ for Lupus I, III, and IV, respectively. The similar ranges spanned by the temperature and mass distributions of prestellar cores in the three Lupus clouds allow us to merge the three samples, and improve the statistical relevance of subsequent analysis.

In Fig. 11, we show the core mass function (CMF) of the total sample of Lupus prestellar cores and that of the robust subsample. A change of the slope of the distribution is present around $0.7 M_{\odot}$, and, in particular the distribution of the robust prestellar cores, becomes almost flat between $0.1 M_{\odot}$ (the completeness limit) and $0.7 M_{\odot}$, with a small peak around 0.2 $0.3 M_{\odot}$. This shape resembles that of the CMF derived from the Herschel data in Taurus (Marsh et al. 2016), a cloud quite similar to Lupus in terms of both mass range and total number of prestellar cores. The evidence of a possible flattening or break near a mass of about $0.5 M_{\odot}$ is also found in the dense cores mass functions derived from ground-based submillimetre surveys (e.g. Motte et al. 1998; Alves et al. 2007; Enoch et al. 2008). The CMFs derived within the HGBS consortium for Aquila and Taurus are well described by log-normal functions (Könyves et al. 2015; Marsh et al. 2016). The log-normal fit to the Lupus CMF of the robust sample, for core masses above the estimated completeness limit $\sim 0.1 M_{\odot}$, is shown in Fig. 11. The best-fit function has a central mass of $(0.25 \pm 0.04) M_{\odot}$ and a standard deviation of $0.55 \pm 0.04$, the same values of the lognormal parameters of the stellar initial mass function (IMF) for multiple systems with masses between 0.12 and $1 M_{\odot}$ (Chabrier 2005). The $\mathrm{K}-\mathrm{S}$ test performed on the cumulative distribution confirms that the observed CMF has a log-normal form with

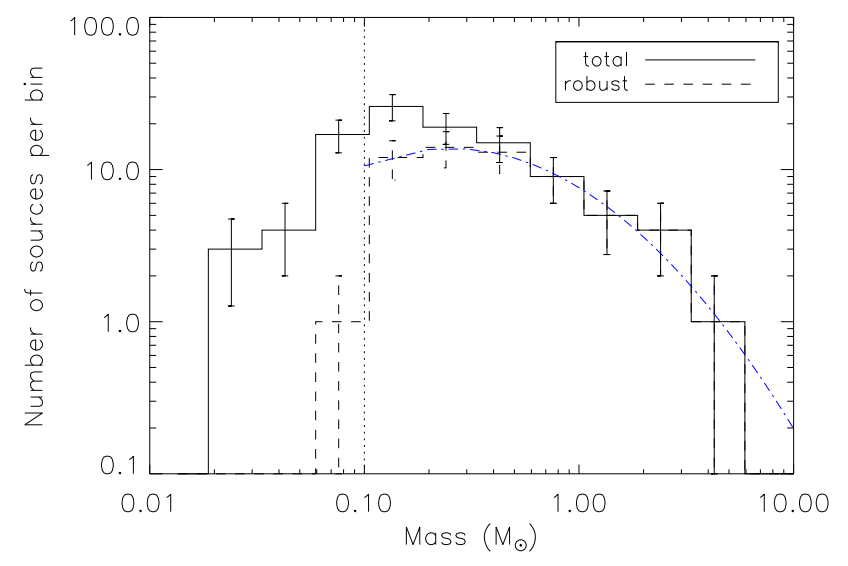

Fig. 11. Core mass function of the total sample of prestellar cores detected in the three Lupus clouds (solid line) and that of the robust sub-sample (dashed line). The dashed-dotted line is the log-normal fit, with central mass of $(0.25 \pm 0.04) M_{\odot}$ and a standard deviation of $0.55 \pm 0.04$. The vertical line indicates the completeness limit of the prestellar cores sample.

$99 \%$ of probability. We recall that, for the high mass tail of the distribution, the statistics are quite poor and the uncertainties on the mass estimate are large; therefore, the real shape of the $\mathrm{CMF}$ for masses larger than $1 M_{\odot}$ cannot be robustly constrained.

The similarity of the shape of the CMF and IMF suggests that the prestellar cores identified in the Herschel catalogue may evolve into single stars on a one-to-one basis. Under this hypothesis, the scaling factor in the mass axis between the two distributions is interpreted in terms of star formation efficiency $\epsilon$, where $M_{\text {star }}=\epsilon M_{\text {core }}$, and it can be estimated from the ratio of the peak of the two distributions. For the Lupus complex, the real peak of the CMF is difficult to define since the histogram of the robust prestallar cores sample is almost flat between 0.1 and $0.7 M_{\odot}$. However, if we consider the peak of the log-normal best fit that has the same value of the Chabrier (2005) IMF, we found that the star formation efficiency might be extremely high, close to $100 \%$, much higher than the $\sim 40 \%$ found in Aquila (Könyves et al. 2015). This result is confirmed also if we compare the Herschel CMF with the distribution of the masses of a sample of class II YSOs in Lupus (Alcalá et al. 2017): the range of the stellar masses are similar to the ones of the Herschel prestellar cores and the peak of the stellar masses distribution is between 0.2 and $0.3 M_{\odot}$. A star formation efficiency close to $100 \%$ is, however, difficult to justify from a physical point of view since the accretion phase of a protostar is associated with significant mass loss through jet and outflow. Probably, we are systematically underestimating the mass of the Herschel prestellar cores. Of all the sources of uncertainty of our prestellar core mass estimate described in Sect. 4.1, the only ones that can produce a systematic shift towards lower masses are the distance and the dust opacity law. If we assume as distance for all the three clouds the upper limit of the distance estimates, namely $d=200 \mathrm{pc}$ (see Sect. 1), the correspondent CMF would peak at $\sim 0.48 M_{\odot}$ with a resulting star formation efficiency of $\sim 50 \%$, more similar to that found in Aquila. However, the new Gaia DR 2 distances point towards a distance closer to $150 \mathrm{pc}$ for the three clouds, rather than 200 pc. More likely, the opacity law we adopted (see Sect. 2) might be not fully appropriate for the low-mass regime of the Lupus cores. Indeed, in Paper I we have already discussed that $\kappa_{300}=0.1 \mathrm{~cm}^{2} \mathrm{~g}^{-1}-$ the standard value of the dust opacity at the reference wavelength assumed in the HGBS consortium - could be too high for the $\mathrm{H}_{2}$ column density range of the Lupus clouds 
and that halving the opacity would reconcile our column density maps to the visual extinction maps. Similarly, reducing the $\kappa_{300}$ by a factor of 2 would produce a CMF almost flat between 0.2 and $0.6 M_{\odot}$. In this case, the shape of CMF is still compatible with the log-normal form with $88 \%$ probability and the best-fit function has a central mass of $(0.34 \pm 0.05) M_{\odot}$, corresponding to $\epsilon \gtrsim 60 \%$, and a standard deviation of $0.41 \pm 0.05$.

\subsection{YSOs/protostars}

Herschel instruments are particularly sensitive to protostars in the younger evolutionary stages, namely Class 0 and I objects, still embedded in substantial dusty envelopes responsible for the FIR emission. In contrast, they can miss the most tenuous, evolved YSOs where the envelope starts to dissipate. Therefore, Herschel data make possible the identification and correct classification of Class 0 and Class I objects more efficiently than previously, but YSOs/protostars catalogues based on only Herschel data can be largely incomplete for Class II and III objects. Despite this shortcoming, the Herschel catalogue is a vital complement to previous YSOs catalogues of the region, in particular that based on Spitzer-c2d data (Merin et al. 2008), because the Herschel maps cover a larger area of sky with respect to the Spitzer maps and because it provides FIR fluxes not available in other catalogues. Indeed, the addition of the Herschel FIR fluxes to the previously known NIR-MIR SEDs is important to estimate the properties of the possible circumstellar envelopes. Such info significantly improves the evolutionary classifications of objects based on their SED shapes, especially for the younger protostars.

Some of the more evolved objects (Class II and III) in our catalogue lack excess emission at MIR wavelengths (typically around 8-24 $\mu \mathrm{m}$ ), but present considerable emission at longer wavelengths. This kind of object, originally discovered by Strom et al. (1989) with IRAS data, evolved YSOs with transitional disks, that are optically thick and gas-rich protoplanetary disks with astronomical unit-scale inner disk clearings or radial gaps. The union of the MIR and Herschel FIR data is a valid tool for detecting such objects and in our catalogue we find several candidates. Three of them (HGBS_YSO-J160711.5390347, HGBS_YSO-J160854.5-393743, and HGBS_YSOJ161051.5-385314) have been previously classified as transitional disks by Bustamante et al. (2015). Eleven other objects in our catalogue (HGBS_YSO-J153927.9-344616, HGBS_YSO_ J154512.8-341729, HGBS_YSO-J160709.9-391102, HGBS YSO-J160822.4-390445, HGBS_YSO-J160825.7-390600, HGBS_YSO-J160829.6-390309, HGBS_YSO-J160836.1392300, HGBS YSO-J160948.5-391116, HGBS YSO-J160002. 5-422216, HGBS_YSO-J160044.6-415530, HGBS_YSOJ160329.2-414001) were also analysed by Bustamante et al. (2015) and though not confirmed as transitional disk candidates, they were classified simply as objects with infrared excesses higher than the median value. Our SED fitting results (see Table 2) confirm these sources as Class II objects with a disk where the detected infrared emission can be ascribed to a residual envelope. Finally, three objects in our YSOs/protostars catalogue, namely HGBS_YSO-J153640.0-3421145, HGBS_YSO-J160830.7382826, HGBS_YSO-J155730.4-421032, from the shape of their SED, are new good candidate YSOs with transitional disks, not present in the list of objects analysed by Bustamante et al. (2015).

We classified the evolutionary state of the YSOs/protostars of the Herschel catalogue by using two indicators: the $\alpha$ index, as used in the Spitzer-c2d catalogue, and the SED fitting. With the latter, we can estimate the presence of three key components of the early phase of the star formation, namely the envelope, the bipolar cavity opened by the outflow, and the disk, whose relevance changes during the evolutionary process. In general, we found that the evolutionary class indicated by the two methods is in agreement (see Table 2). In Appendix C, however, we discuss the few cases where we do not find full agreement.

\section{Summary and conclusions}

The nearby star-forming clouds of the Lupus complex have been mapped in five photometric bands at 70, 160, 250, 350, and $500 \mu \mathrm{m}$ with Herschel photometric instruments within the HGBS key project. In this paper, we presented the catalogues of dense cores and YSOs/protostars in Lupus I, III, and IV, compiled from the extraction of compact sources in the five Herschel maps. Two dedicated procedures for sources extraction and selection have been applied, optimised for dense cores and protostars, respectively. A total of 532 dense cores and 38 YSOs/protostars have been identified in the maps. Catalogues, listing their measured properties, namely position, flux density and size at each band, are supplied. In addition, we also provide catalogues of the physical properties listing mass, temperature, radius, column density, and average volume density for the dense cores and bolometric luminosity, FIR luminosity, $\alpha$ spectral index, and evolutionary class for the protostars.

The physical properties of the starless dense cores were estimated by fitting the observed SEDs with a grey-body function. The comparison of the measured mass with the BE mass was used to select a sample of 103 candidates prestellar cores, complete down to masses of $\sim 0.1 M_{\odot}$. Almost all the prestellar cores are associated with the brightest filamentary structures of the clouds, confirming one of the main results of the Herschel photometric surveys of star-forming regions, that filaments are the preferred place for the formation of the dense condensations that will evolve in new stars. Conversely, we found that only about one third of the starless cores and YSOs/protostars are associated with filaments. In Lupus I, we found a higher level of clumpiness of cores, possibly due to its younger evolutionary stage in terms of star formation activity.

In Lupus, we found robust prestellar core candidates even in regions with background column density lower than that measured in other star-forming regions so far. In particular, we found that $90 \%$ of prestellar cores lie on a background higher than a visual extinction threshold of about $2 \mathrm{mag}$. In other clouds, most of which have typical prestellar core masses higher than the Lupus ones, values of this background threshold from 5 to 9 mag were found. The lower limit of the column density background is often interpreted as a column density threshold for the star formation, however the large variability of its value found in several star-forming regions indicates that this limit should be interpreted more as a higher probability to find prestellar cores in the parts of the molecular clouds with column densities above it rather than a stringent limit under which the star formation is inhibited. Moreover, its value might depend on the local properties of the single star-forming cloud such as the strength of its magnetic field, its radiation field, and its typical non-thermal velocity dispersion.

Overall, the analysis of the prestellar cores catalogue indicates that the physical properties of the Lupus sample are similar to those of other regions studied with Herschel. One peculiar characteristic is the significant number of prestellar condensations with very low mass $\left(\lesssim 0.2 M_{\odot}\right)$, in line with the low column density of its ISM and with the low mass of its main sequence 
stars. We derived the CMF of the prestellar cores total sample. The CMF of the robust prestellar cores has a log-normal form with a peak between 0.2 and $0.3 M_{\odot}$; this implies a very high efficiency in the conversion of the prestallar core mass into the stellar mass that could be an indication that we are underestimating the prestallar core masses of the Lupus clouds. Anyway, the Lupus CMF shape is consistent with previous findings for other star-forming clouds and with the stellar systems IMF.

Noticeably, in the Lupus star-forming regions, we found that the majority of filaments that contain prestellar cores have average masses per unit length below the maximum value possible for thermal support. For such low-column-density filaments, the mass per unit length averaged along the full filament is not a good parameter for identifying star-forming filaments.

The physical properties of the YSOs/protostars were estimated by building their SEDs over a wide wavelength range, from NIR to FIR wavelengths and fitting the SED with a set of theoretical models. With the SED fitting, we gauged the possible presence around the protostar of three key components of the early phase of star formation: the envelope, the bipolar cavity opened by the outflow, and the disk, whose relevance changes during the evolutionary process. We provide the range of input parameters of the models with good fit to the observed SEDs. We estimated the evolutionary status of the YSOs/protostars using two indicators: the $\alpha$ spectral index and the result of the SED fitting. For about $70 \%$ of the objects, the evolutionary stages derived with the two methods agree.

The Herschel catalogue of YSOs/protostars, although incomplete for objects in the later evolutionary stages, adds high value to previous catalogues because the addition of FIR fluxes to the previously known NIR-MIR SEDs largely improves the identification and evolutionary classification of the younger protostars based on their SED shape, allowing estimates to be made of the properties of the possible circumstellar envelope. Moreover, our Herschel YSOs/protostars catalogue of the Lupus I, III, and IV regions includes objects not present in previous catalogues and identifies new candidates of Class 0 objects and YSOs with transitional disks.

Acknowledgements. K.L.J.R. acknowledges financial support by the Italian Ministero dell'Istruzione, Università e Ricerca, through the grant Progetti Premiali 2012-iALMA (CUP C52I13000140001). N.S. acknowledges support by the French ANR and the German DFG through the project GENESIS (ANR-16-CE92-0035-01/DFG1591/2-1). P.P. acknowledges support from Fundação para a Ciência e a Tecnologia of Portugal (FCT) through national funds (UID/FIS/04434/2013) and by FEDER through COMPETE2020 (POCI01-0145-FEDER-007672) and also by the fellowship SFRH/BPD/110176/2015 funded by FCT (Portugal) and POPH/FSE (EC). This work has received support from the European Research Council under the European Union's Seventh Framework Programme (ERC Advanced Grant Agreements No. 291294 "ORISTARS").

\section{References}

Alcalá, J. M., Manara, C. F., Natta, A., et al. 2017, A\&A, 600, A20

Alves, J., Lombardi, M., \& Lada, C. J. 2007, A\&A, 462, L17

André, P., Ward-Thompson, D., \& Barsony, M. 2000, Protostars and Planets IV eds. V. Mannings, A. P. Boss, \& S. S. Russell (Tucson: University of Arizona Press), 59

André, P., Men'shchikov, A., Bontemps, S., et al. 2010, A\&A, 518, L102

André, P., Di Francesco, J., Ward-Thompson, D., et al. 2014, Protostars and Planets VI, eds. H. Beuther, R. S. Klessen, C. P. Dullemond, \& T. Henning (Tucson: University of Arizona Press), 27

Arzoumanian, D., André P., Didelon P., et al. 2011, A\&A, 529, L6

Arzoumanian, D., André, P., Peretto, N., \& Könyves, V. 2013, A\&A, 553, A119
Balog, Z., Müller, T., Nielbock, M., et al. 2014, Exp. Astron., 37, 129 Bendo, G. J., Griffin, M. J., Bock, J. J., et al. 2013, MNRAS, 433, 3062 Benedettini, M., Pezzuto, S., Burton, M., et al. 2012, MNRAS, 419, 238 Benedettini, M., Schisano, E., Pezzuto, S., et al. 2015, MNRAS, 453, 2036 (Paper I)

Bernard, J.-P., Paradis, D., Marshall, D.J., et al. 2010, A\&A, 518, L88

Bohlin, R. C., Savage, B. D., \& Drake, J. F. 1978, ApJ, 224, 132

Bresnahan, D., Ward-Thompson, D., Kirk, J. M., et al. 2018, A\&A, 615, A125

Bustamante, I., Merín, B., Ribas, Á., et al. 2015, A\&A, 578, A23

Cambrésy, L. 1999, A\&A, 345, 965

Chabrier, G. 2005, The Initial Mass Function 50 Years Later, eds. E. Corbelli, F. Palla, \& H. Zinnecker, Astrophys. Space Sci. Lib., 327, 41

Comerón, F. 2008, Handbook of Star Forming Regions: Vol II. The Southern Sky, ed. B. Reipurth (San Francisco: ASP), 295

Crapsi, A., Caselli, P., Walmsley, M. C., \& Tafalla, M. 2007, A\&A, 470, 221

Elia, D., Molinari, S., Fukui, Y., et al. 2013, ApJ, 772, 45

Enoch, M. L., Evans, II, N. J., \& Sargent, A. I. 2008, ApJ, 684, 1240

Evans, II, N. J., Dunham, M. M., \& Jørgensen, J. K. 2009, ApJS, 181, 321

Foster, J.B., Rosolowsky, E. W., Kauffmann, J., et al. 2009, ApJ, 696, 298

Gaczkowski, B., Roccatagliata, V., Flaischlen, S., et al. 2017, A\&A, 584, A36

Galli, D., Walmsley, M., \& Gonçalves, J. 2002, A\&A, 394, 275

Galli, P. A. B., Bertout, C., Teixeira, R., \& Ducourant, C. 2013, A\&A, 558, A77

Giannini, T., Elia, D., Lorenzetti, D., et al. 2012, A\&A, 539, A156

Greene, T. P., Wilking, B. A., André, P., Young, E. T., \& Lada, C. J. 1994, ApJ, 434, 614

Griffin, M. J., Abergel, A., Abreu, A., et al. 2010, A\&A, 518, L3

Hatchell, J., Richer, J. S., Fuller, G. A., et al. 2005, A\&A, 440, 151

Heitsch, F., \& Hartmann, L. 2008, ApJ, 689, 290

Hildebrand, R. H. 1983, QJRAS, 24, 267

Hughes, J., Hartigan, P., Krautter, J., \& Kelemen, J. 1994, AJ, 108, 1071

Inutsuka, S., Inoue, T., Iwasaki, K., \& Hosokawa, T. 2015, A\&A, 580, A49

Johnstone, D., Di Francesco, J., \& Kirk, H. 2004, ApJ, 611, 45

Keown, J., Di Francesco, J., \& Kirk, H. 2017, ApJ, 850, 3

Kirk, H., Johnstone, D., \& Di Francesco, J. 2006, ApJ, 646, 1009

Kirk, J. M., Ward-Thompson, D., Palmeirim, P., et al. 2013, MNRAS, 432, 1424

Könyves, V., André, P., Men'shchikov, A., et al. 2015, A\&A, 584, A91

Lada, C., \& Wilking, B. A. 1984, ApJ, 287, 610

Lada, C., Lombardi, M., \& Alves, J. F. 2010, ApJ, 724, 687

Lada, C., Lombardi, M., Roman-Zuniga, C., Forbrich, J., \& Alves, J. F. 2013, ApJ, 778, 133

Liseau, R., Lorenzetti, D., Nisini, B., Spinoglio, L., \& Moneti, A. 1992, A\&A, 265, 577

Lombardi, M., Lada, C., \& Alves, J. F. 2013, A\&A, 559, A90

Luri, X., Brown, A. G. A., Sarro, L. M., et al. 2018, A\&A, 616, A9

McKee, C. F. 1989, ApJ, 345, 782

Marsh, K. A., Kirk, J. M., André, P., et al. 2016, MNRAS, 459, 342

Menten, K. M., Reid, M. J., Forbrich, J., \& Brunthaler, A. 2007, A\&A, 474, 515

Men'shchikov A. 2013, A\&A, 560, A63

Men'shchikov A. 2016, A\&A, 593, A71

Men'shchikov, A., André, P., Didelon, P., et al. 2012, A\&A, 542, A81

Merin, B., Jørgensen, J., Spezzi, L., et al. 2008, ApJS, 177, 551

Molinari, S., Schisano, E., Faustini, F., et al. 2011, A\&A, 530, A133

Mortier, A., Oliveira, I., \& van Dishoeck, E. F. 2001, MNRAS, 418, 1194

Motte, F., André, P., \& Neri, R. 1998, A\&A, 336, 150

Mowat, C., Hatchell, J., Rumble, D., et al. 2017, MNRAS, 467, 812

Nielbock, M., Müller, T., Klass, U., et al. 2013, Exp. Astron., 36, 631

Onishi, T., Mizuno, A., Kawamura, A., Ogawa, H., \& Fukui, Y. 1998, ApJ, 502, 296

Padoan, P., Juvela, M., Goodman, A. A., \& Nordlund, Å. 2001, ApJ, 553, 227

Palmeirim, P. P., André, P., Kirk, J., et al. 2013, A\&A, 550, A38

Pilbratt, G. L., Riedinger, J. R., Passvogel, T., et al. 2010, A\&A, 518, L1

Poglitsch, A., Waelkens, C., Geis, N., et al. 2010, A\&A, 518, L2

Polychroni, D., Schisano, E., Elia, D., et al. 2013, ApJ, 777, L33

Rayner, T. S. M., Griffin, M. J., Schneider, N., et al. 2017, A\&A, 607, A22

Rygl, K. L. J., Benedettini, M., Schisano, E., et al. 2013, A\&A, 549, L1

Robitaille, T. P. 2017, A\&A, 600, A11

Roy, A., André, P., Palmeirim, P., et al. 2014, A\&A, 562, A138

Schisano, E., Rygl, K. L. J., Molinari, S., et al. 2014, ApJ, 791, 27

Strom, S. E., Edwards, S., \& Strom, K. M. 1989, The Formation and Evolution of Planetary Systems, eds. H. A. Weaver \& L. Danly (Cambridge, UK: Cambridge University Press), 91

Tafalla, M., Myers, P. C., Caselli, P., Walmsley, C. M., \& Comito, C. 2002, ApJ, 569,815

Tafalla, M., Myers, P. C., Caselli, P., \& Walmsley, C. M. 2004, A\&A, 416, 191

Tothill, N. F. H., Löhr, A., Parshley, S. C., et al. 2009, ApJS, 185, 98

Ulrich, R. K. 1976, ApJ, 210, 377 


\section{Appendix A: Dense cores and YSOs/protostars catalogues identified in Lupus I, III, and IV based on Herschel data}
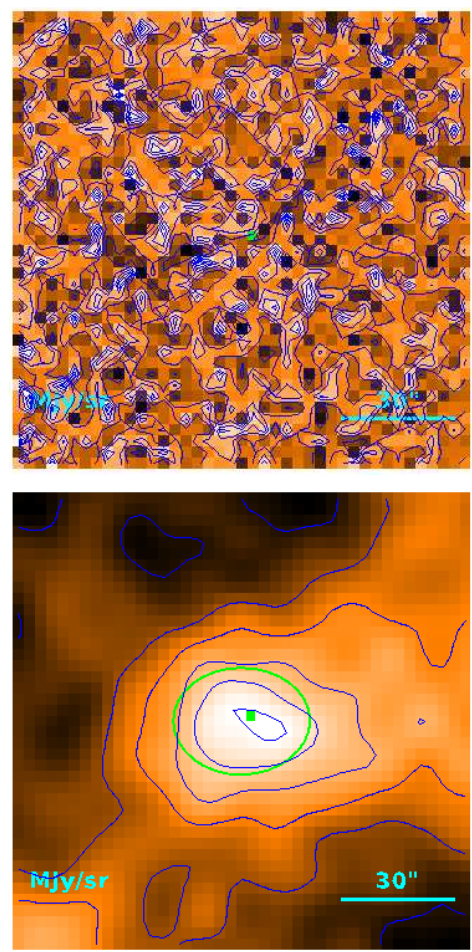
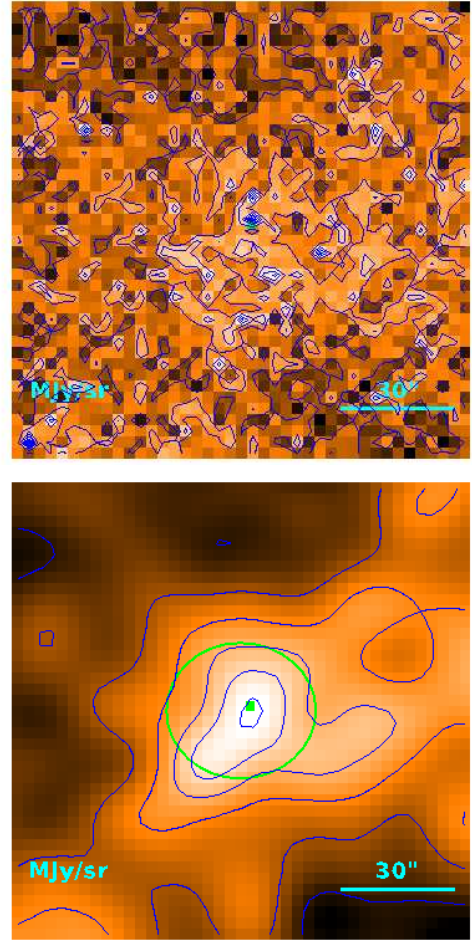
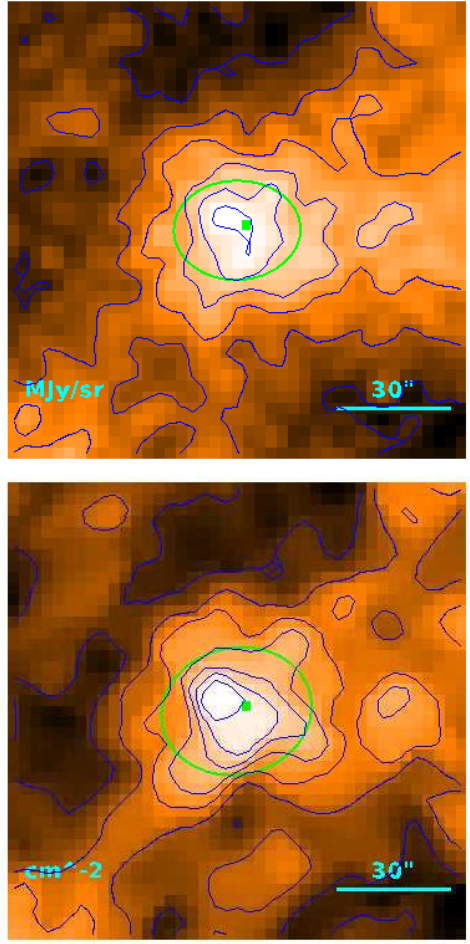

Fig. A.1. $2^{\prime} \times 2^{\prime}$ cuts of the Herschel images at 70,160, 250,350, and $500 \mu \mathrm{m}$ and high-resolution column density map (in this order) centred on the HGBS-J153809.7-34074 dense core of our catalogue that has been classified as an unbound starless core. Green ellipses represent the estimated FWHM sizes of the core at each wavelength. Similar images for all the dense cores in our catalogues are provided at http://gouldbelt-herschel.cea.fr/archives.

We extracted dense cores and YSOs/protostars in the five Herschel maps of the Lupus I, III, and IV molecular clouds. As examples, in Figs. A.1-A.3, we show $2^{\prime} \times 2^{\prime}$ maps at $70,160,250,350$, and $500 \mu \mathrm{m}$ and the high-resolution column density map for an unbound starless core, a prestellar core, and a protostar, respectively. Similar images for all the sources in our catalogues are provided at http:// gouldbelt-herschel. cea. fr/archives. The complete catalogues of dense cores and YSOs/protostars as well as the physical catalogues are available at the CDS. Here, we provide a few lines of the catalogues to show the entries we provide. In Table A.1, we list the source name and position together with flux density measurements and FWHM at each band as measured in the maps. The physical properties of dense cores, both unbound and prestellar, are derived from SED fitting at the Herschel bands performed as explained in Sect. 4.1. In Fig. A.4, we show the SED best fit for two sources, a similar image for all the dense cores in our catalogues is provided at http://gouldbelt-herschel. cea.fr/archives. For the unbound starless core HGBS-J153809.7-34074 (left panel of Fig. A.4), it is possible to find a theoretical SED that not only fits very well the fluxes in the SPIRE bands, but is also perfectly compatible with the flux upper limits in the PACS bands. For the prestellar core HGBS-J154024.0-33373, however, the $160 \mu \mathrm{m}$ upper limit is clearly incompatible with the SPIRE fluxes (see the central panel of Fig. A.4). No theoretical SED has a shape that can fit both the observed fluxes and upper limits at the same time. Clearly, it is highly debatable that one upper limit counts more than three fluxes, and in any case the upper limit also has its own uncertainty.

In building the SEDs, we put an upper limit to the flux at a given wavelength when the source is not visible in any clean single scales from getsources (Men'shchikov et al. 2012). In this case the flux derived at the measurement stage is very uncertain. When an upper limit is too stringent and does not agree with the fluxes at the other bands, as for source HGBS-J154024.033373, we put the SIG_MON parameter of the getsources output to a very low value, that is $9.999 \mathrm{E}-30$. When this happen the ellipse that shows the geometrical properties of the source at the given band is shown in red, as shown in Fig. A.2, to warn the reader that the flux is very uncertain. Since each SIG_MON provides the weight of the respective measurement in the SED fitting procedure, and since for valid fluxes this value is always higher than a few units, it is clear that a flux that has a weight 30 or more orders of magnitude smaller than the other fluxes does not play any role in determining the bestfit SED. This change, however, has the important consequence that the upper limit does not longer constrain the shape of the SED.

The result of using a flux with a very low weight instead of an upper limit is shown in the right panel of Fig. A.4. Now the best-fit SED reproduces very well the fluxes at all wavelengths. We note that the agreement with the $160 \mu \mathrm{m}$ datum is by chance, probably because even if getsources was not able to detect the source, it could nonetheless make a valid estimate of its flux. In other cases, however, the datum at $160 \mu \mathrm{m}$ is not compatible with 
M. Benedettini et al.: A catalogue of dense cores and young stellar objects in the Lupus complex
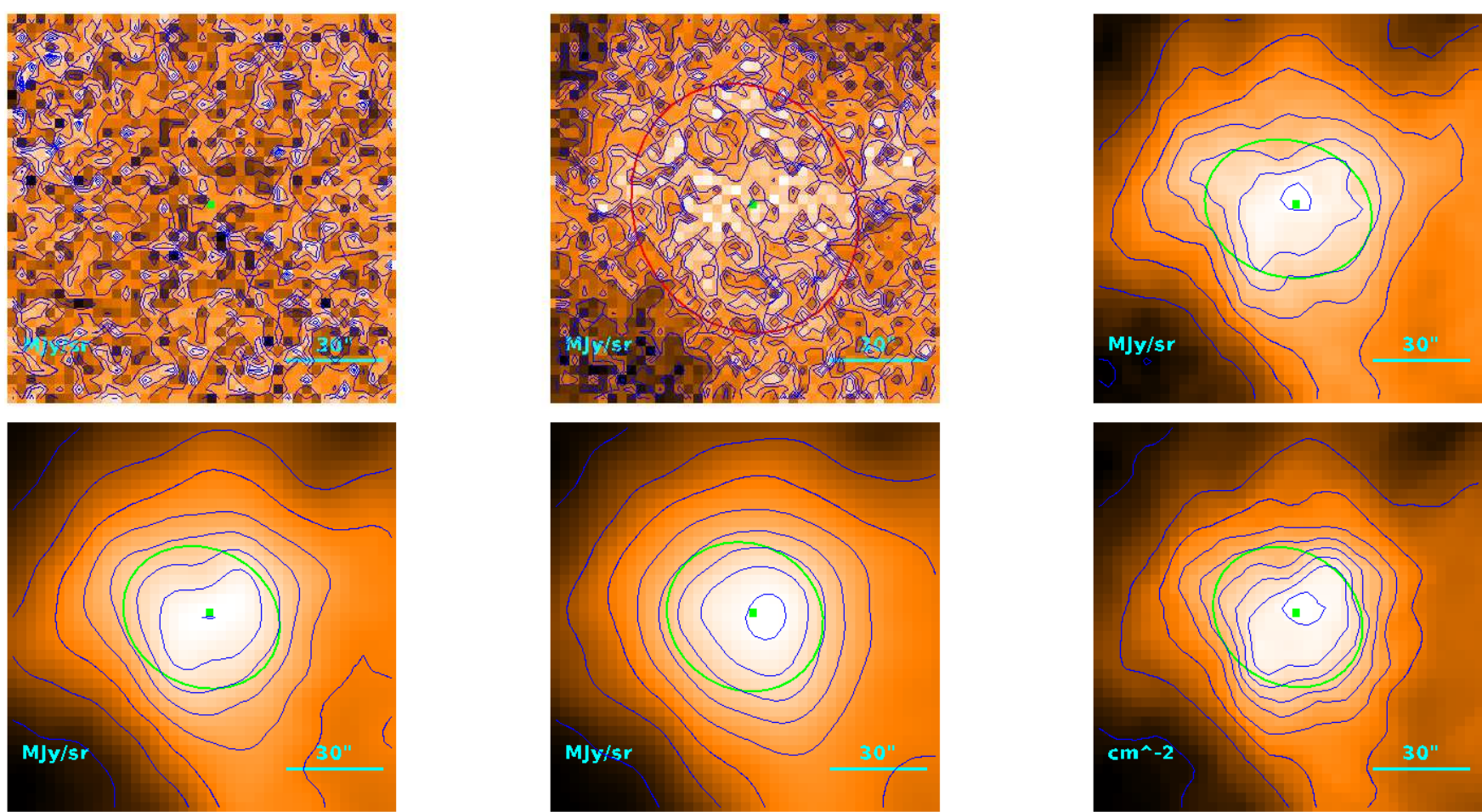

Fig. A.2. Same as Fig. A.1 for the HGBS-J154024.0-33373 dense core of our catalogue that has been classified as a prestellar core. Red ellipse indicates an uncertain flux estimate (see text for details).
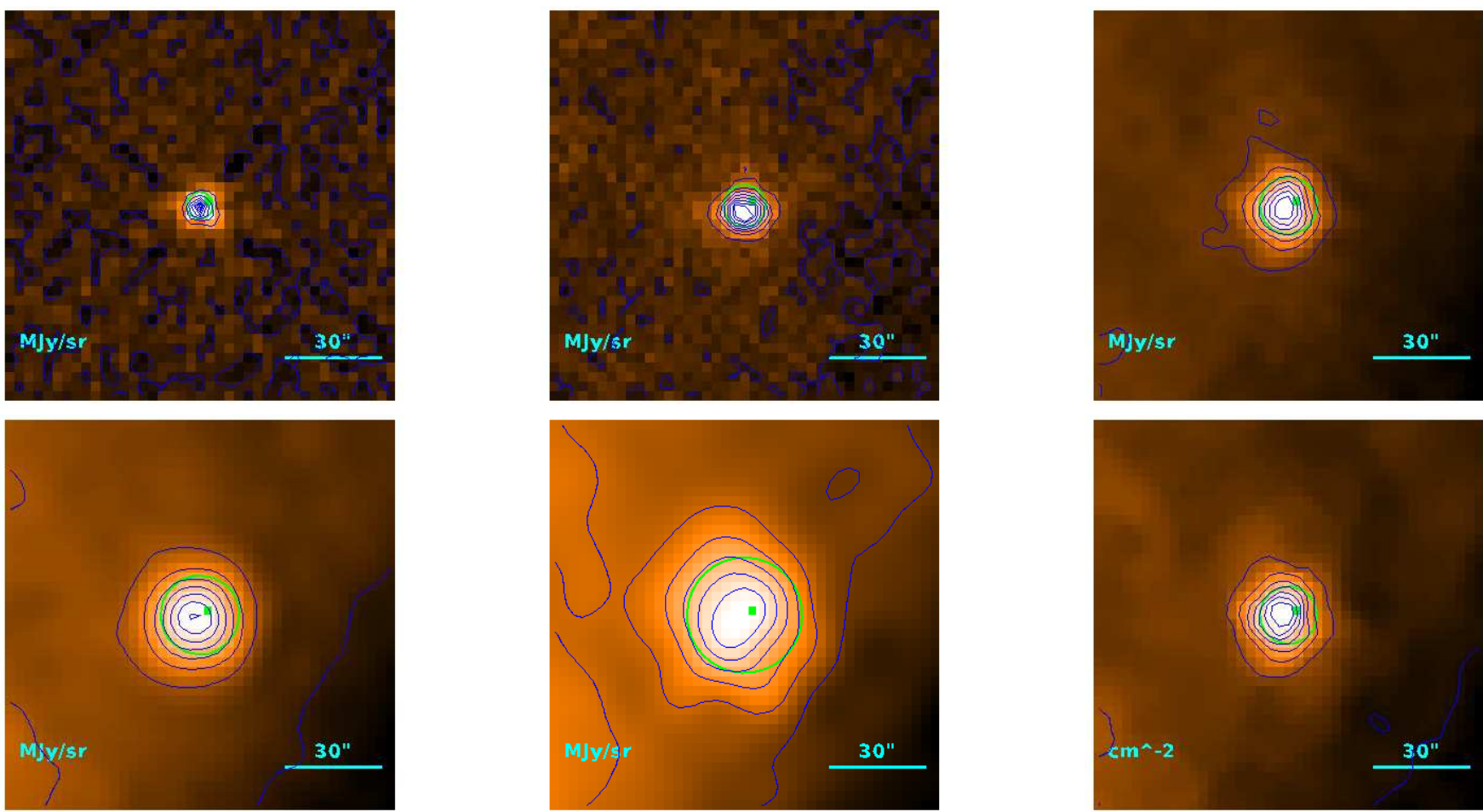

Fig. A.3. Same as Fig. A.1 for the protostar HGBS_YSO-J160044.6-415530. Similar images for all the YSOs/protostrs in our catalogues are provided at the following URLs: https://owncloud.ia2.inaf.it/index.php/s/TiJtzW5gWo3YwKA, https://owncloud. ia2. inaf.it/index.php/s/LHfkSLFOpI63icB, https://owncloud.ia2 . inaf.it/index. php/s/TMnYDhv9uj 1mNHg, for Lupus I, III and IV, respectively. 

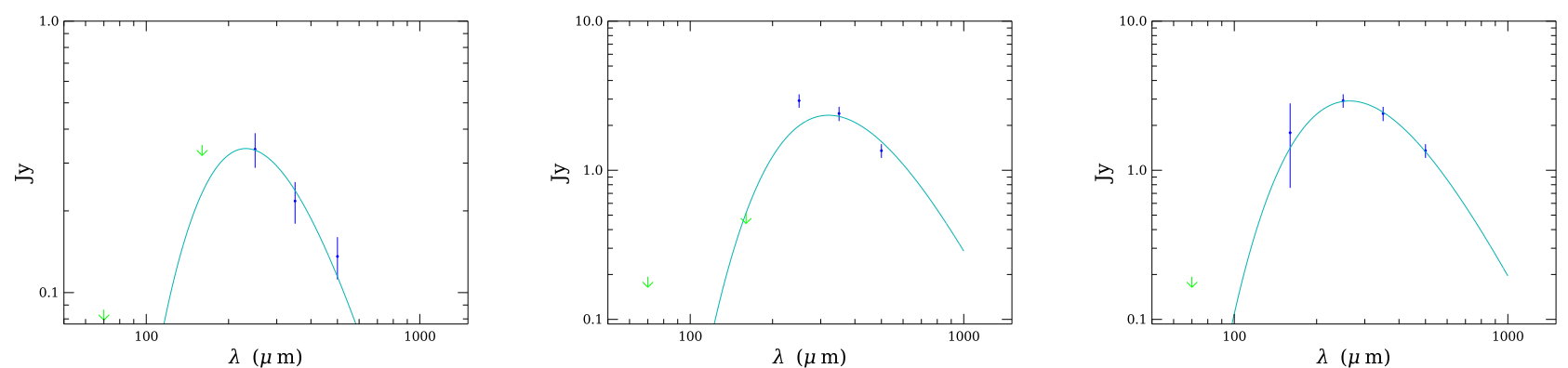

Fig. A.4. Grey-body fit to the SED at the Herschel bands for sources HGBS-J153809.7-34074 (left panel) and HGBS-J154024.0-33373 (central and right panels) of the dense cores catalogue. Figures of the observed SED and the grey-body fit for all the dense cores in our catalogues are provided at http://gouldbelt-herschel.cea.fr/archives. Upper limits are indicated with green arrows. Source HGBS-J154024.0-33373 was not detected at $160 \mu \mathrm{m}$. In the central panel, we show the best SED fit that respects the upper limit at this wavelength: we cannot find any grey-body model that fits the SPIRE fluxes and the PACS upper limits at the same time. In the right panel, the $160 \mu$ m upper limit is relaxed (see text in Appendix A) and flux with a very low weight is used in the fitting. Now we can find a very good fit to the observed SED.

the best-fit SED, because its weight in the fitting procedure is so low that it is as if that flux does not count.

The fact that the grey-body model now fits well the data has, of course, important consequences on the physical parameters of the source. In general, we found that the temperature derived when we impose that the fit is consistent with the $160 \mu \mathrm{m}$ upper limit is around 7-9 K, much lower than the average temperature of about $12 \mathrm{~K}$, showing that this upper limit cannot be used. In the particular case of source HGBS-J154024.0-33373, considering the upper limit at $160 \mu \mathrm{m}$ results in $T=9.05_{-0.03}^{+0.04} \mathrm{~K}$ and $M=0.337 \pm 0.023 M_{\odot}$. When the upper limit is not taken into account (again stressing that this does not mean that we are using the $160 \mu \mathrm{m}$ flux, but that we are not considering it as a stringent upper limit), the parameters are $T=10.99_{-0.07}^{+0.08} \mathrm{~K}$ and $M=0.159 \pm 0.010 M_{\odot}$. The latter temperature is now much more similar to the average value and the mass is less than $50 \%$ the value found when considering the upper limit.

The results of the SED fits for all sources, namely physical radius, mass, SED dust temperature, peak column density at the resolution of the $500 \mu \mathrm{m}$ data, average column density, peak volume density, and average density are given in Table A.2 for the dense cores catalogue.

\section{Appendix B: YSOs SED fitting}

The Robitaille (2017) synthetic SEDs are divided into 18 sets of models where the central source may or may not be associated with a disk, a circumstellar envelope, a bipolar cavity and an ambient medium. Each set of models represents a different combination of these components and each component is described by several input parameters. The detailed description of the physics of each component and its physical parameters can be found in Robitaille (2017), and here we briefly report the list of the free input parameters of the models. All free parameters are uniformly sampled within an allowed range. The central star, present in all models, is a spherical source and is defined by a stellar radius $R_{\star}$ and an effective temperature $T_{\star}$. The passive flared disk has a density distribution that is defined by the disk dust mass $M_{\text {disk }}$, the inner and outer radii $R_{\min }^{\text {disk }}$ and $R_{\max }^{\text {disk }}$, respectively, the surface density radial exponent $p_{\text {disk }}$, the disk flaring exponent $\beta_{\text {disk }}$, and the disk scale height $h_{100 \mathrm{AU}}$. Two types of envelopes are included in the models: the first is a spherically symmetric power-law envelope, and the second is a rotationally flattened envelope as defined by Ulrich (1976). For both envelope components, the envelope is truncated at the inner radius $R_{\mathrm{min}}^{\mathrm{env}}$, and extends all the way to the edge of the grid. The free parameters are the envelope density scaling $\rho_{0}^{\text {env }}$, the radial exponent $\gamma_{\mathrm{env}}$ in the case of the power-law envelope, and the centrifugal radius $R_{\mathrm{c}}$ in the case of the Ulrich envelope. The bipolar cavity, present only in models where an envelope component is also present, is defined by the power-law exponent of the cavity opening $c_{\text {cav }}$, the opening angle $\theta_{0}^{\text {cav }}$, and the density inside the cavity is set to $\rho_{0}^{\text {cav }}$ or the envelope density, whichever is lowest. In several models an ambient medium is added with dust density of $\rho_{\mathrm{amb}}=10^{-23} \mathrm{~g} \mathrm{~cm}^{-3}$ and temperature $T_{\mathrm{amb}}=10 \mathrm{~K}$. Each model was computed for nine viewing angles randomly sampled between $0^{\circ}$ and $90^{\circ}$.

To determine which model best represents the observed SED, we follow the fitting procedure described in Robitaille (2017). We define a "good" model as one with

$\chi^{2}-\chi_{\text {best }}^{2}<9 n_{\text {data }}$,

where $\chi_{\text {best }}^{2}$ is the lowest $\chi^{2}$ among all model sets and $n_{\text {data }}$ is the number of data points in the observed SED. We then define the probability of a model set $P$ as the ratio between the number of good models $N_{\text {good }}$ and the total number of models with the set $N$

$P=\frac{N_{\text {good }}}{N}$.

Finally, we assign a score to each set of models as the ratio of $P$ to the mean of the $P$ values for all model sets. A higher relative score indicates a more likely model, therefore we consider as the best model set the one with the highest score.

In Table B.1, for each object of our Herschel YSOs/protostars catalogue, we report the model set with the highest score, using the same nomenclature of Robitaille (2017), and we indicate which of the four model components are included in the best models set. In addition, for all the objects we provide, in electronic form, files with the range of physical parameters of all the good models within the best model set and figures of the synthetic SED of the models with the best $\chi^{2}$ for all 18 sets of models of Robitaille (2017). This material is provided at the following URLs:

https://owncloud.ia2.inaf.it/index.php/s/ aVkJ07nYKVj9FlD,

https://owncloud.ia2.inaf.it/index.php/s/ SxkNLXMi9iMIFYd,

https://owncloud.ia2.inaf.it/index.php/s/ zUvSRoLLX2Ckt08,

https://owncloud.ia2.inaf.it/index.php/s/ CDQF jFPPJ eXu2v1, 


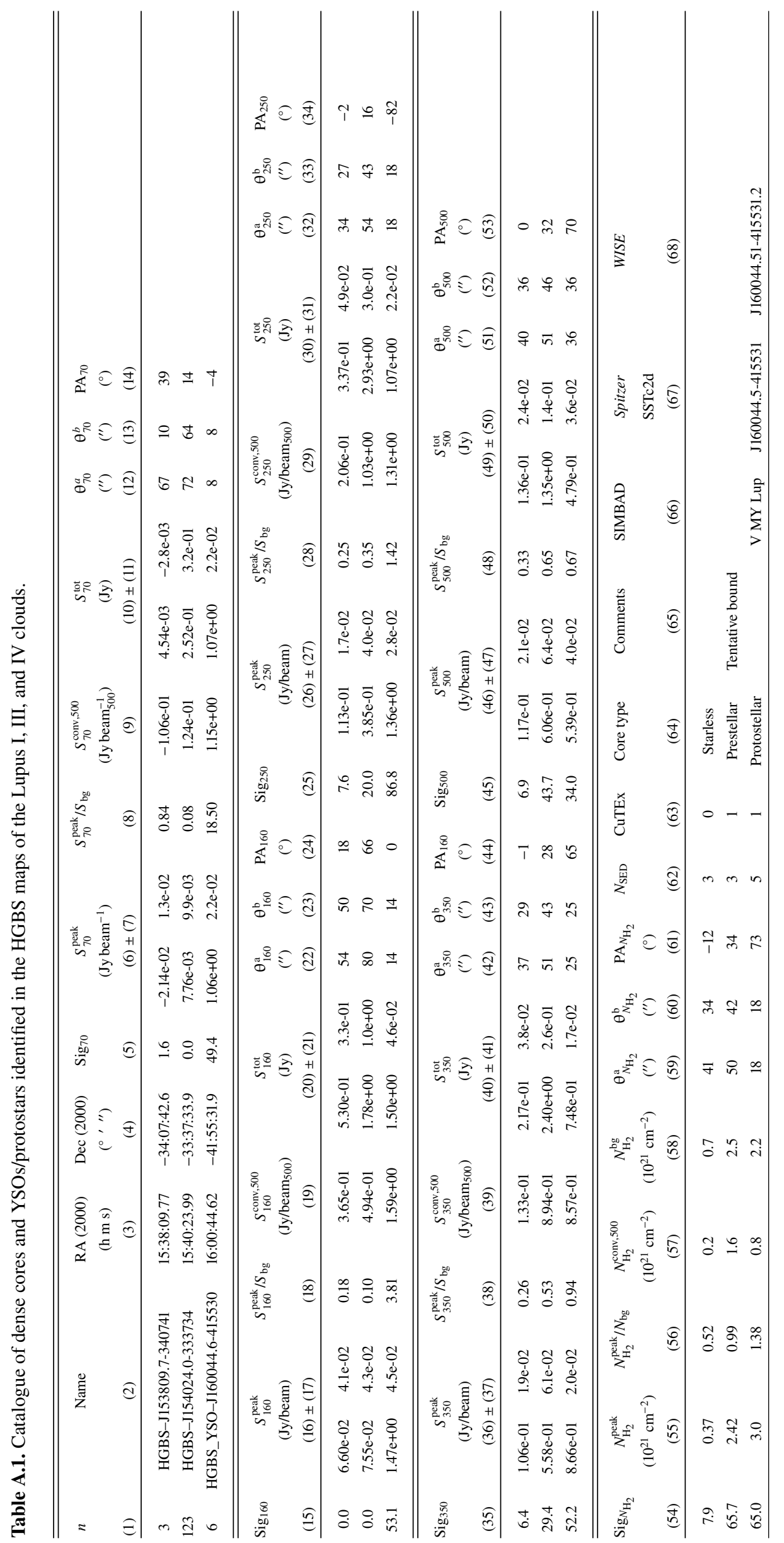

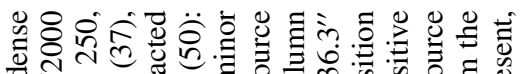

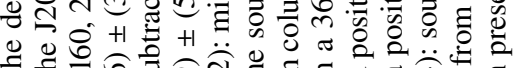

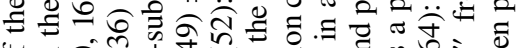

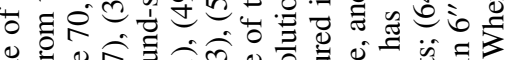

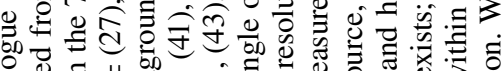

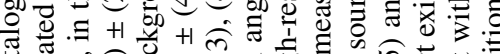

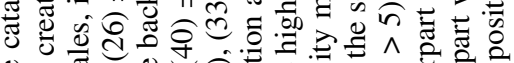

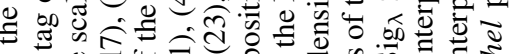
क्ष 0 कo

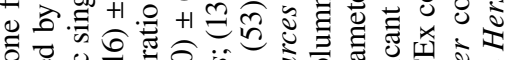
o

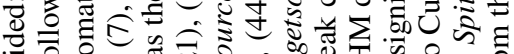
可

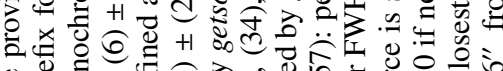

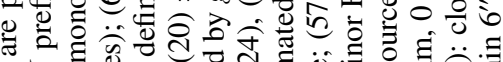

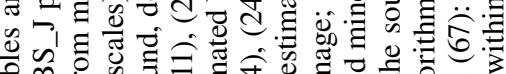

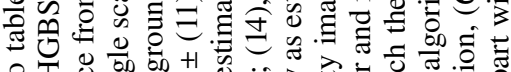

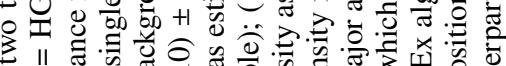

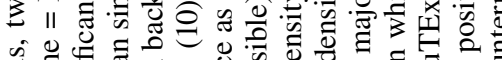

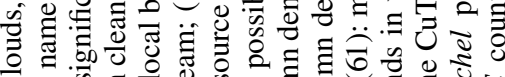
o o 5 .

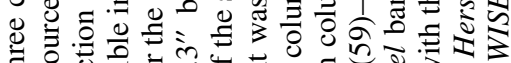

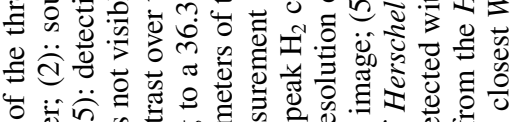

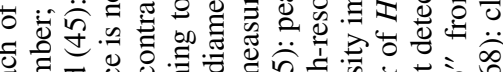

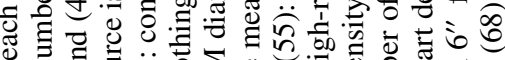
等

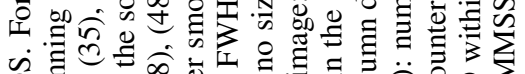
के o d 300

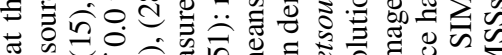

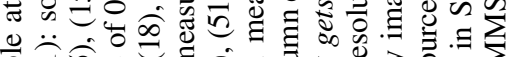

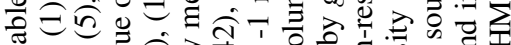

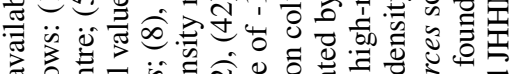

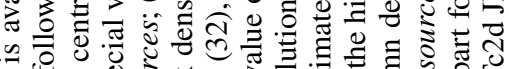

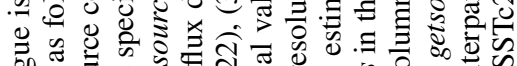

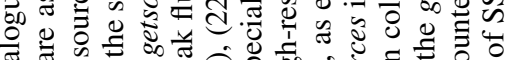

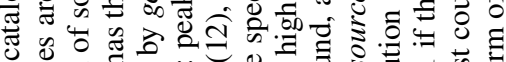

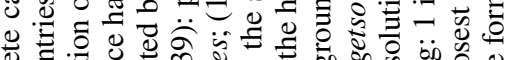

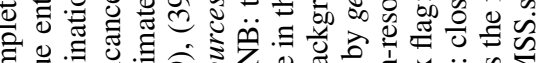

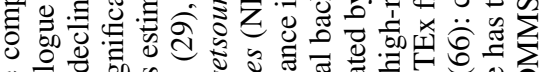
o ن क

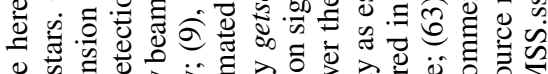

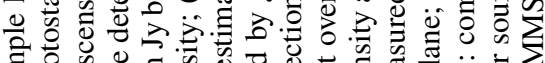

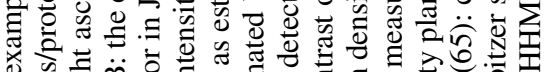
ऽ。

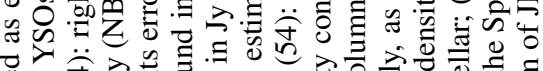

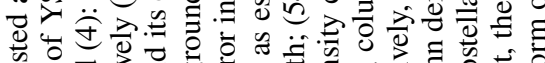

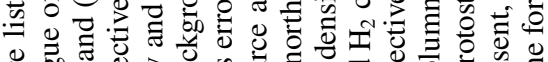

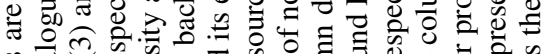
o 屈

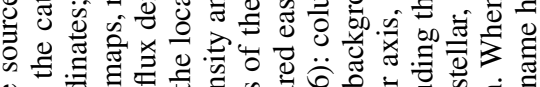

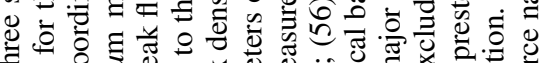

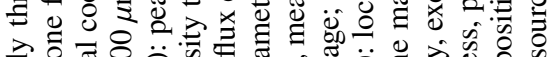

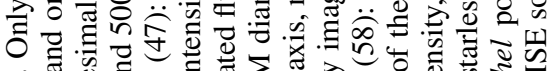

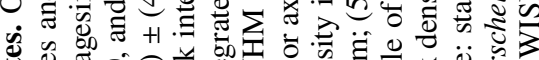

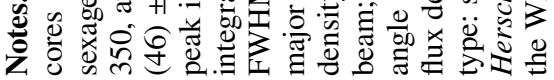




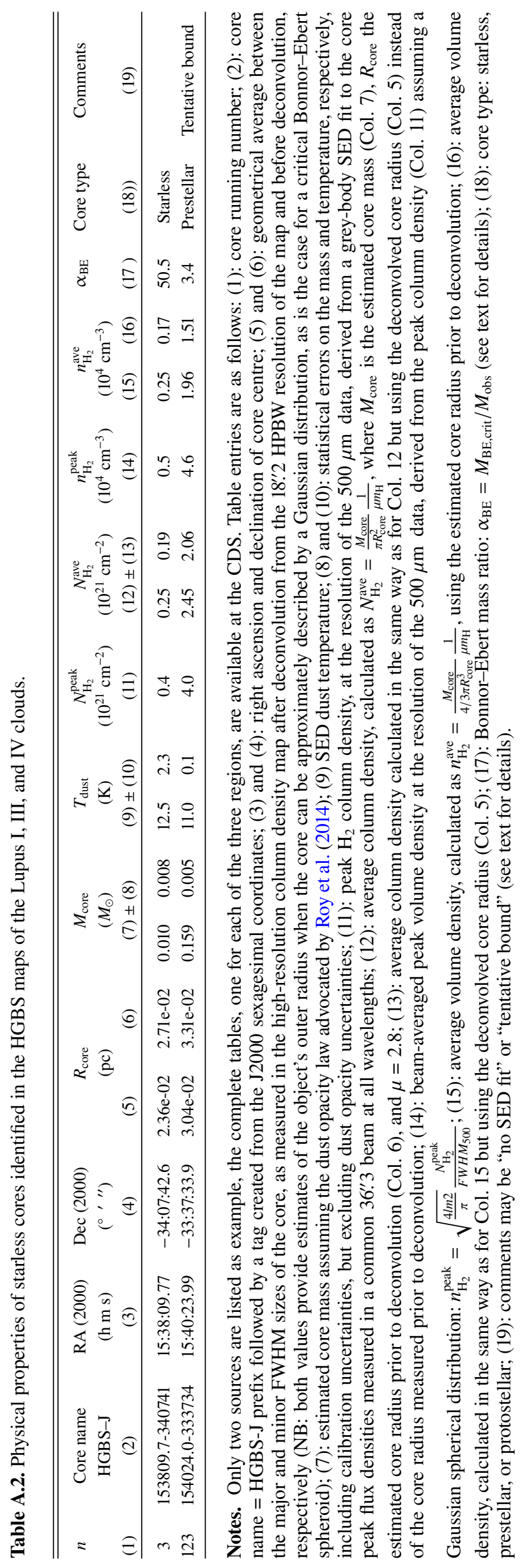


M. Benedettini et al.: A catalogue of dense cores and young stellar objects in the Lupus complex

Table B.1. List of the most probable set of models for the YSOs/protostars of our catalogue fitted with the Robitaille (2017) model.

\begin{tabular}{|c|c|c|c|c|c|c|c|c|}
\hline Source & $n_{\text {data }}$ & Best model set & Score & Disk & Envelope & Cavity & Ambient & Inner radius \\
\hline \multicolumn{9}{|l|}{ LUPUS I } \\
\hline HGBS_YSO-J153640.0-342145 & 11 & spubhmi & 18.0 & Yes & Ulrich & Yes & Yes & Variable \\
\hline HGBS_YSO-J153927.9-344616 & 18 & spubhmi & 14.0 & Yes & Ulrich & Yes & Yes & Variable \\
\hline HGBS_YSO-J154011.3-351522 & 8 & $\mathrm{sp}-\mathrm{h}-\mathrm{i}$ & 10.8 & Yes & No & No & No & Variable \\
\hline HGBS_YSO-J154017.6-324649 & 9 & spubhmi & 11.5 & Yes & Ulrich & Yes & Yes & Variable \\
\hline HGBS_YSO-J154051.6-342102 & 12 & spubhmi & 18.0 & Yes & Ulrich & Yes & Yes & Variable \\
\hline HGBS_YSO-J154302.3-340908 & 13 & s-pbsmi & 18.0 & No & Power-law & Yes & Yes & $R_{\text {sub }}$ \\
\hline HGBS_YSO-J154512.8-341729 & 15 & $\mathrm{sp}-\mathrm{s}-\mathrm{i}$ & 18.0 & Yes & No & No & No & $R_{\text {sub }}$ \\
\hline HGBS_YSO-J154529.8-342339 & 18 & spubsmi & 12.0 & Yes & Ulrich & Yes & Yes & $R_{\text {sub }}$ \\
\hline HGBS_YSO-J154644.6-343034 & 12 & spubsmi & 8.2 & Yes & Ulrich & Yes & Yes & $R_{\text {sub }}$ \\
\hline \multicolumn{9}{|l|}{ LUPUS III } \\
\hline HGBS_YSO-J160500.9-391301 & 5 & $\mathrm{sp}-\mathrm{s}-\mathrm{i}$ & 18.0 & Passive & No & No & No & $R_{\text {sub }}$ \\
\hline HGBS_YSO-J160708.4-391407 & 18 & spu-smi & 8.0 & Passive & Ulrich & No & Yes & $R_{\text {sub }}$ \\
\hline HGBS_YSO-J160709.9-391102 & 18 & spubsmi & 13.5 & Passive & Ulrich & Yes & Yes & $R_{\mathrm{sub}}$ \\
\hline HGBS_YSO-J160711.5-390347 & 18 & spubhmi & 12.0 & Passive & Ulrich & Yes & Yes & Variable \\
\hline HGBS_YSO-J160822.4-390445 & 18 & spubhmi & 18.0 & Passive & Ulrich & Yes & Yes & Variable \\
\hline HGBS_YSO-J160825.7-390600 & 17 & $\mathrm{sp}-\mathrm{s}-\mathrm{i}$ & 10.3 & Passive & No & No & No & $R_{\text {sub }}$ \\
\hline HGBS_YSO-J160829.6-390309 & 15 & s-pbhmi & 18.0 & No & Power-law & Yes & Yes & Variable \\
\hline HGBS_YSO-J160830.7-382826 & 11 & spubhmi & 18.0 & Passive & Ulrich & Yes & Yes & Variable \\
\hline HGBS_YSO-J160836.1-392300 & 18 & $\mathrm{sp}-\mathrm{smi}$ & 18.0 & Passive & No & No & Yes & $R_{\text {sub }}$ \\
\hline HGBS_YSO-J160854.5-393743 & 13 & s-pbsmi & 18.0 & No & Power-law & Yes & Yes & $R_{\text {sub }}$ \\
\hline HGBS_YSO-J160901.8-390511 & 15 & spubsmi & 6.0 & Passive & Ulrich & Yes & Yes & $R_{\text {sub }}$ \\
\hline HGBS_YSO-J160917.9-390453 & 14 & spubhmi & 18.0 & Passive & Ulrich & Yes & Yes & Variable \\
\hline HGBS_YSO-J160948.5-391116 & 17 & $\mathrm{sp}-\mathrm{s}-\mathrm{i}$ & 18.0 & Passive & No & No & No & $R_{\text {sub }}$ \\
\hline HGBS_YSO-J161051.5-385314 & 18 & spubsmi & 8.4 & Passive & Ulrich & Yes & Yes & $R_{\text {sub }}$ \\
\hline \multicolumn{9}{|l|}{ LUPUS IV } \\
\hline HGBS_YSO-J155641.9-421925 & 11 & spubsmi & 18.0 & Yes & Ulrich & Yes & Yes & $R_{\text {sub }}$ \\
\hline HGBS_YSO-J155730.4-421032 & 11 & s-u-hmi & 14.4 & No & Ulrich & No & Yes & Variable \\
\hline HGBS_YSO-J155746.6-423549 & 12 & spubsmi & 9.0 & Yes & Ulrich & Yes & Yes & $R_{\text {sub }}$ \\
\hline HGBS_YSO-J155916.5-415712 & 12 & $\mathrm{sp}-\mathrm{smi}$ & 9.0 & Yes & No & No & Yes & $R_{\text {sub }}$ \\
\hline HGBS_YSO-J160044.6-415530 & 18 & spubhmi & 18.0 & Yes & Ulrich & Yes & Yes & Variable \\
\hline HGBS_YSO-J160115.5-415233 & 18 & spubhmi & 7.2 & Yes & Ulrich & Yes & Yes & Variable \\
\hline HGBS_YSO-J160234.6-421129 & 14 & spubhmi & 6.0 & Yes & Ulrich & Yes & Yes & Variable \\
\hline HGBS_YSO-J160329.2-414001 & 13 & spubsmi & 6.0 & Yes & Ulrich & Yes & Yes & $R_{\text {sub }}$ \\
\hline HGBS_YSO-J160403.0-413427 & 7 & sp-h-i & 18.0 & Yes & No & No & No & Variable \\
\hline HGBS_YSO-J160913.7-414430 & 8 & sp-s-i & 18.0 & Yes & No & No & No & $R_{\text {sub }}$ \\
\hline HGBS_YSO-J160956.3-420834 & 6 & $\mathrm{sp}-\mathrm{s}-\mathrm{i}$ & 18.0 & Yes & No & No & No & $R_{\text {sub }}$ \\
\hline HGBS_YSO-J161301.6-415255 & 9 & $\mathrm{sp}-\mathrm{h}-\mathrm{i}$ & 18.0 & Yes & No & No & No & Variable \\
\hline
\end{tabular}

Notes. The names of the sets of models are those defined in Robitaille (2017). For each model, the presence of disk, envelope, cavity, and ambient medium is indicated. For the envelope, two possible profiles, spherically symmetric power-law or Ulrich (1976) type, are specified. For all models that contain at least a disk, an envelope, or an ambient medium, the inner radius is set to the same value for all components, and is either set to $R_{\text {sub }}$ (the dust sublimation radius) or is variable in the range $R_{\text {sub }}-1000 R_{\text {sub }}$.

https://owncloud.ia2.inaf.it/index.php/s/ cSp9bk0ZYgx5wuk,

https://owncloud.ia2.inaf.it/index.php/s/ opcGd8WGN3NVdoS.

\section{Appendix C: Notes on particular YSOs}

In this appendix we discuss the result of the SED fitting for those YSOs of our catalogue that have a poor fit and those where we find some discrepancies with the spectral index classification.

For HGBS_YSO-J154017.6-324649, we find only a counterpart in WISE but not in Spitzer-IRAC and 2Mass. It has an $\alpha$ index of a Class I object but with such significant emission at longer wavelengths that the $L_{\mathrm{smm}} / L_{\mathrm{bol}}$ ratio is typical of a Class 0 object. The SED fit indicates the presence of a little disk and a robust dusty envelope with a cavity. Its location in a very low column density region and the fact that it is not visible $500 \mu \mathrm{m}$ with Herschel makes it very unlikely that it is a Class 0 objects and favours the interpretation that it is a more evolved object.

For source HGBS_YSO-J154529.8-342339, the NIR-MIR part of its SED is typical of a Class II object and indeed it is well fitted by a disk. However, it has been associated with a FIR Herschel source, making its $L_{\mathrm{smm}} / L_{\mathrm{bol}}$ suggestive of a Class 0 object. Its SED model fails to match the FIR part of its SED. In fact, looking at the Herschel maps, it is likely that the quoted fluxes at 350 and $500 \mu$ mare contaminated by close-by sources.

For source HGBS_YSO-J160500.9-391301 we have only five photometric points and the best $\chi^{2}$ SED model is able to 
reproduce only three of the points, therefore the result is really uncertain.

For source HGBS_YSO-J160708.4-391407, the $\alpha$ index indicates a Class Flat object but the $L_{\mathrm{smm}} / L_{\mathrm{bol}}$ ratio is typical of a Class 0 object. However, this source is well detected at NIR wavelengths up to the $J$ band. Moreover, the best-fit model of the full SED indicates the presence of a disk with a tenuous envelope, favouring for this object the interpretation of a more evolved Class Flat rather than Class 0.

For source HGBS_YSO-J160709.9-391102, the $\alpha$ index indicates a Class II object while the SED fitting gives as model with the highest score one with a very low mass disk of $2.7 \times 10^{-08} M_{\odot}$, seen edge on, with a quite dense circumstellar envelope with a cavity. However, a similar good fit can be also obtained with a model with only a circumstellar disk with mass of $2.8 \times 10^{-02} M_{\odot}$ and an inclination angle of $65^{\circ}$. This second model is compatible with the Class II classification.

HGBS_YSO-J160829.6-390309 is a well known T-Tauri star. It is quite luminous at MIR wavelengths up to $160 \mu \mathrm{m}$ that make its $\alpha$ index typical of the Class I objects, however, it is not detected at the Herschel-SPIRE bands $(\lambda \geq 250 \mu \mathrm{m})$. It has been classified as higher than the median infrared excess in Bustamante et al. (2015; see Sect. 6.6), and the best set of models is that with e circumstellar envelope and a cavity. However, also models with disk and wihout envelope, compatible with Class II classification, give good fit to the observed SED.

HGBS_YSO-J155641.9-421925 is the brightest source in Lupus IV and it is a well-known Herbig Ae star (HD 142527). The SED best fit reproduces well its MIR-FIR SED but fails to fit the NIR data.
HGBS_YSO-J155730.4-421032 is a Class II object. The most probable set of models, however, indicates only the presence of an envelope, though models with cavity and disk can also fit well the observed SED.

For source HGBS_YSO-J160115.5-415233, the $\alpha$ index indicates a Class Flat object but the $L_{\mathrm{smm}} / L_{\mathrm{bol}}$ ratio is typical of a Class 0 object. In this case, the SED fit is consistent with the earlier evolutionary stage, indicating the presence of a massive envelope $\left(\rho_{0}^{\text {env }}=1.1 \times 10^{-20} \mathrm{~g} \mathrm{~cm}^{-3}\right)$ and a meagre disk $\left(M_{\text {disk }}=4.3 \times 10^{-8} M_{\odot}\right)$. An additional indication in favour of the youth of this source is the ratio between the column density of $\mathrm{HC}_{3} \mathrm{~N}$ and $\mathrm{NH}_{3}$, reported in Benedettini et al. (2012), that is similar to what is observed towards the Class 0 object HGBS_YSO-J160917.9-390453 in Lupus III and is higher than typical values found in more evolved protostars. The majority of the observational evidence therefore indicates that this source is a good Class 0 protostar candidate.

HGBS_YSO-J160403.0-413427 and HGBS_YSOJ160956.3-420834 have strange SEDs lacking the MIR fluxes, indicating a possible accidental association between the NIR and FIR data. Moreover, their $\alpha$ indexes cannot be defined since not enough data are available. In both cases, the SED fits indicate that they are evolved objects, possibly Class II, with a disk and without envelope. HGBS_YSO-J160956.3-420834 is associated with a known star (HD 142527)

HGBS_YSO-J161301-415255 is a Class III object. The best fit reproduces well its NIR-MIR SED but fails to reproduce the fluxes between 24 and $160 \mu \mathrm{m}$, indicating the possible presence of another (younger) source close to the YSO. The position of this object is not covered by the Herschel-SPIRE maps. 